$11^{\text {th }}$ National Scientifc Congress SPML, 5-6 April 2019, Coimbra, Portugal • https://doi.org/10.1515/ cclm-2019-1173

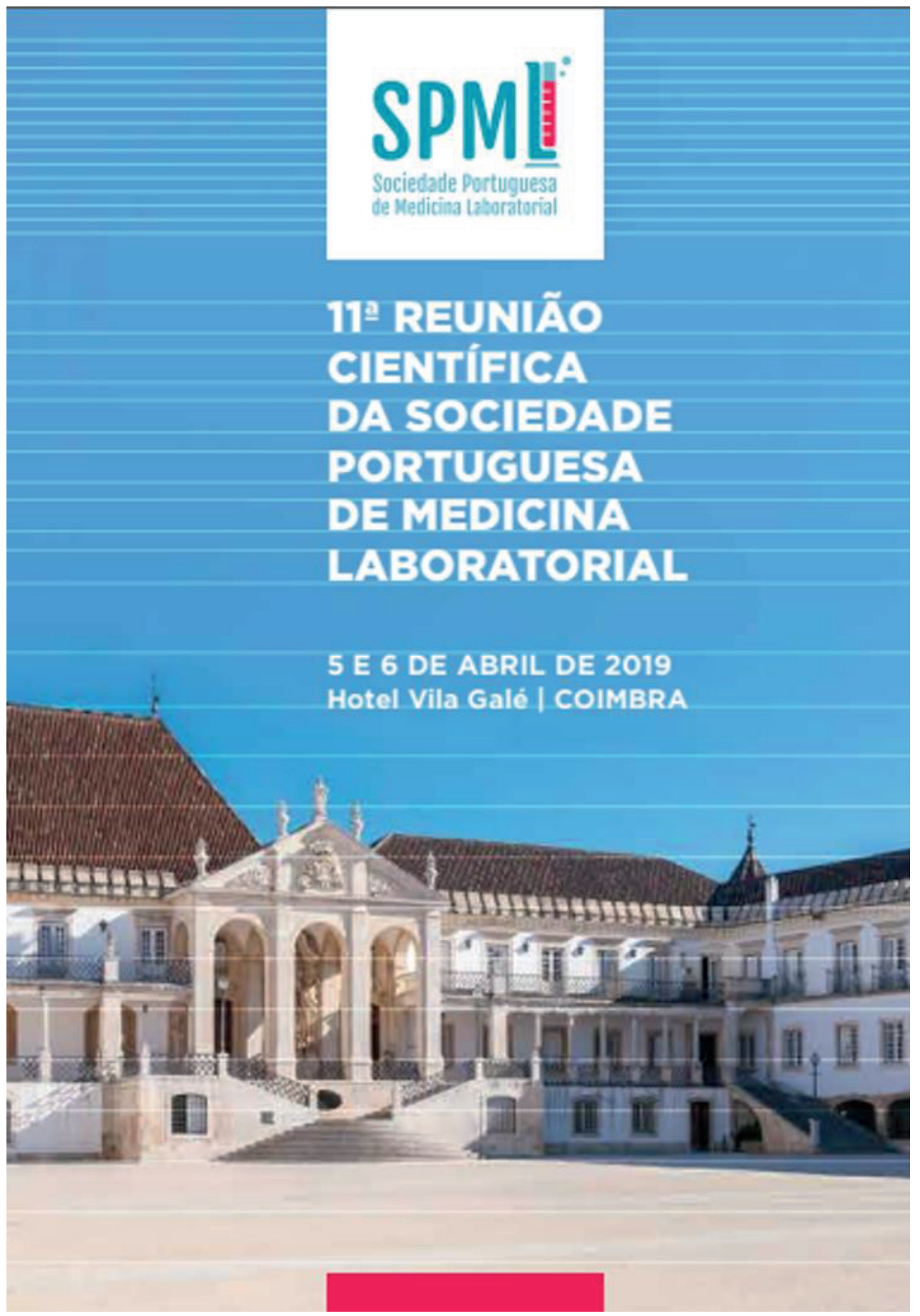




\title{
SCIENTIFIC COMISSION
}

\author{
Adriana Pedrosa \\ Ana Paula Castro \\ Ana Paula Faria \\ Catarina Chaves \\ Elsa Gonçalves \\ Eulália Costa \\ Fátima Vale \\ Fernando Rodrigues \\ Helena Brízido \\ Henrique Reguengo \\ João Faro Viana \\ João Tiago Guimarães \\ Jorge Nunes de Oliveira \\ José Cortez \\ Luísa Espinhaço \\ Ma José Teles \\ Ma Luís Queirós \\ Rosário Luís \\ Rui Farinha
}


P01

MANTLE CELL LYMPHOMA AND CHRONIC LYMPHOCYTIC LEUKEMIA/ SMALL LYMPHOCYTIC LYMPHOMA: A CASE REPORT

Carlos Martins ${ }^{1}$; Joana Sevilha ${ }^{2}$, Sara Braga ${ }^{3}$; Cátia Rodrigues ${ }^{3}$; Cátia Mendes ${ }^{3}$; Carlos Palmeira ${ }^{3}$; M. Emília de Sousa ${ }^{3}$; Inês Godinho ${ }^{3}$; Ana Pires ${ }^{3}$; Carla Azevedo ${ }^{3}$; Ângelo Martins ${ }^{4}$; Gabriela Martins ${ }^{3}$

${ }^{1}$ Serviço de Patologia Clínica, Centro Hospitalar Vila Nova de Gaia/Espinho, EPE

${ }^{2}$ Serviço de Patologia Clínica, Centro Hospitalar do Tâmega e Sousa, EPE; ${ }^{3}$ Serviço de Imunologia, Instituto Português de Oncologia do Porto Francisco Gentil, EPE

${ }^{4}$ Serviço de Onco-Hematologia, Instituto Português de Oncologia do Porto Francisco Gentil, EPE

Introduction: The concomitant identification of Mantle cell lymphoma (MCL) or Chronic lymphocytic leukemia/Small lymphocytic lymphoma (CLL/SLL) with other lymphoid tumors has been described. However the concomitant occurrence of both $M C L$ and $C L L / S L L$ in the same patient as described in this case report is rare.

\section{Clinical case:}

A 73-year-old man was admitted in our hospital with lymphocytosis and thrombocytopenia, without B symptoms, an ultrasound image compatible with splenomegaly and monoclonal peak IgG/L.

Immunophenotypic characterization (by multiparametric flow cytometry) and genetic study were made through peripheral blood sampling. Regarding the immunophenotype analysis, an antibody panel was used to study lymphoproliferative syndromes, according to Euroflow protocols, as well as the proliferative index evaluation through the study of DNA content (percentage of S-phase cells). The flow cytometric characterization revealed the presence of two different populations of pathological B lymphocytes.

One population had a $31.53 \%$ of pathological cells, with phenotype CD20++ CD79b+ CD5+/++ CD10- CD11c-/+ (5\%) CD19++ CD20++ CD23- CD79b+ CD200- HLADR+ IgM+, restriction of lambda light chains and showed a low proliferative index of $0.10 \%$. These results were compatible with mantle cell lymphoma. The second population presented $17 \%$ of pathological cells, with phenotype CD20+ CD79+ CD5+/++ CD10CD11c-/+ CD19+ CD20+ CD23+ CD79b- CD200+ HLADR+ IgM- restriction of kappa light chains and a low proliferative index of $0.18 \%$, compatible with chronic lymphocytic leukemia B-cell type. Genetic study showed two cytogenetically unrelated clones, one with $\mathrm{t}(11 ; 14)$ (q13; q32) translocation and the other with 12 trisomy, making this result compatible with the presence of $\mathrm{MCL}$ and $\mathrm{CLL} / \mathrm{SLL}$.

Discussion: $\mathrm{MCL}$ and $\mathrm{CLL} / \mathrm{SLL}$ share many features and both arise from CD5+ B-cells. Their distinction is critical as MCL is a much more aggressive neoplasm. The flow cytometry immunophenotypic analysis has an important role in this clinical case because it allowed the detection of these two distinct mature B-cell lymphoma, although they share similar morphologic characteristics. These results were then confirmed by cytogenetic results. This diagnosis is important for therapeutic decision making. 
P02

\section{SAMPLE QUALITY ASSESSMENT IN FLOW CYTOMETRY}

Sara Braga, Ana Torgal, João Pedro Barreto, Cátia Mendes, Cátia Rodrigues, Ana Marta Pires, Carla Azevedo, Carlos Palmeira, M. Emília de Sousa, Inês Godinho, Gabriela Martins

Serviço de Imunologia Instituto Português de Oncologia do Porto (IPO), Francisco Gentil, E.P.E

Introduction: Bone marrow (BM) aspirates for Flow Cytometry (FC) analysis are usually obtained from a second aspiration, since the first aspirate is used for morphological assessment. That's why FC samples almost unavoidably contain some blood contamination.

The evaluation of the quality of samples for FC is, therefore, of the greatest importance, to make sure they are of representative value, allowing a more accurate interpretation of the obtained results, especially for Minimal Residual Disease (MRD) quantitation.

Two clinical cases of Multiple Myeloma (MM) are reported where MRD evaluation by FC was negative despite the disease being detected by another method. For evaluation of the haemodilution degree of samples a method described in the literature was used, based on the percentage of plasma cells, CD34+ cells and CD10+ granulocytes: Peripheral Blood Contamination Index $(\mathrm{PBCl})=$ $-3.052+0.065 \times\left(\% \mathrm{CD} 10^{+} \mathrm{G}\right)-0.609 \times\left(\% \mathrm{CD} 34^{+}\right)-2.008 \times(\% \mathrm{PC})$.

$\mathrm{PBCl}>1.2$ discriminates low quality samples.

\section{Case Reports:}

1. MM, IgA kappa, ISS 1 , DS I-A since $11 / 2017$, in complete remission. The second portion of the BM aspirate arrived on the 60th day post-autotransplant for detection of MRD by FC, which revealed no pathological plasma cell ( $>2 \times 10^{6}$ cells were acquired). Immunofixation (IF) revealed an IgA kappa monoclonal band. $\mathrm{PBCl}$ was 1.74.

2. MM, IgG kappa, ISS 2, DS III-A since $12 / 2017$, in partial remission. The second portion of the BM aspirate arrived on the 60th day post-autotransplant for detection of MRD by FC, which revealed no pathological plasma cell ( $>2 \times 10^{6}$ cells were acquired). Electrophoresis (EF) revealed a quantifiable IgG kappa monoclonal peak of 7.37/L. $\mathrm{PBCl}$ was 3.11.

\section{Discussion:}

Both results raised doubts for the Clinical Pathologist since FCM is supposed to be more sensitive than an IF or an EF. That is why it was decided to determine the degree of haemodilution of the samples.

On both cases the samples had $\mathrm{PBCl}>1.2$ and were considered inadequate for the evaluation of MRD, since they underestimated the presence of malignant cells.

This compelled the department to report a measurable criterion of contamination to stimulate colleagues to collect a quality sample for FCM analysis, namely, the $1^{\text {st }}$ tap of the marrow aspirate, as recommended by Euroflow Guidelines.

This method has been implemented as a daily routine since 11/2018. 
P03

\section{PROSTATE HEALTH INDEX IMPROVES PROSTATE CANCER DETECTION}

Manuel M Garrido ${ }^{1}$, Rui M Bernardino ${ }^{2}$, Ruy M Ribeiro ${ }^{3}$, Maria H Pereira ${ }^{4}$, Luís C Pinheiro $^{5}$, João T Guimarães ${ }^{6}$

${ }^{1}$ Department of Clinical Pathology, Central Lisbon University Hospital Center \& Department of Laboratory Medicine, School of Medicine, University of Lisbon, Lisbon, Portugal

2 Department of Urology, Central Lisbon University Hospital Center, Lisbon, Portugal

${ }^{3}$ Biomathematics Laboratory, School of Medicine, University of Lisbon, Lisbon, Portugal

${ }^{4}$ Department of Pathologic Anatomy, Central Lisbon University Hospital Center \& Department of Pathologic Anatomy, Nova Medical School, Lisbon, Portugal

5 Department of Urology, Central Lisbon Hospital Center \& Department of Urology, Nova Medical School, Lisbon, Portugal

${ }^{6}$ Department of Clinical Pathology, Sao Joao Hospital Center; Department of Biomedicine, School of Medicine \& Institute of Public Health, University of Porto, Porto, Portugal

Introduction and Objective: Prostate Specific Antigen (PSA) is not sufficiently specific for prostate cancer ( $\mathrm{PCa})$, therefore, it is important to find new biomarkers that could improve PCa detection. In PCa there is an increase in circulating isoforms of free PSA, such as [-2]proPSA. This marker allows the calculation of the Prostate Health Index (PHI). Our primary objective was to compare the diagnostic performance of $\mathrm{PHI}$, with total PSA (tPSA) and the percentage of free PSA (\% $\mathrm{fPSA}$ ).

Methods: 64 patients with a tPSA between 2 and $10 \mathrm{ng} / \mathrm{mL}$ (Hybritech ${ }^{\circledast}$ calibration) scheduled to undergo a prostate needle biopsy with 12 cores, were enrolled. Exclusion criteria were: previous history of $\mathrm{PCa}$, transuretral ressection of prostate, therapeutic with 5 - $\alpha$-reductase inhibitors and androgens, urinary tract infection and acute bacterial prostatitis. All PSA markers were assayed with the Beckman Coulter ${ }^{\circledR}$ Access ${ }^{\circledR}$ automated immunoassay system. PHI was calculated using the formula ([2]proPSA/free PSA) $\times$ VtPSA. All biopsies were examined by the same pathologist. Patients with high grade intraepithelial neoplasia, intraductal carcinoma or atypical small acinar proliferation, were considered as not having PCa. Statistical analyses, Receiver Operating Characteristic (ROC) curves and t tests, were carried out using $\mathrm{IBM}^{\circledR}$ SPSS $^{\circledR}$ Statistics v. 24.

Results: 30 patients (47\%) had PCa. PHI was significantly higher in the group with PCa (47 vs $29, p=0.001$ ). The area under the ROC curve for PHI was higher than for tPSA and \%fPSA (table 1). 


\begin{tabular}{ll}
\hline & AUC $(95 \% \mathrm{Cl})$ \\
\hline tPSA & $0.594(0.453-0.735)$ \\
\%fPSA & $0.662(0.525-0.798)$ \\
PHI & $0.761(0.645-0.878)$ \\
\hline AUC = area under the curve; $\mathrm{Cl}=$ confidence interval
\end{tabular}

Table 1 - Comparison of AUC between tPSA, \%fPSA and PHI.

$\mathrm{PHI}$ (cutoff $=26$ ) had a sensitivity of $90 \%$ and a specificity of $47 \%$, and its use would allow sparing of 16 unnecessary biopsies (25\%). With tPSA (cutoff $=4 \mathrm{ng} / \mathrm{mL}$ ), only 5 (8\%) biopsies would be avoided; with \%fPSA (cutoff $=25 \%$ ), $7(11 \%)$ would be avoided . $\mathrm{PHI}$ was higher in the group of patients with a total Gleason score (GS) $\geq 7$ (50.7 vs $34.3)$, but not significantly $(p=0.176)$.

Conclusions: PHI shows a better diagnostic performance than tPSA and \% FPSA in men with a serum tPSA level between 2 and $10 \mathrm{ng} / \mathrm{mL}$, allowing to spare $25 \%$ of biopsies. $\mathrm{PHI}$ is higher, but not significantly, in patients with more aggressive forms of PCa $(\mathrm{GS} \geq 7)$. 
P04

APPLICATION AND VALIDATION OF AN EQUATION FOR ADJUSTMENT OF SERUM COPPER TO CERULOPLASMIN AND ITS CLINICAL APPLICABILITY TO WILSON DISEASE

\author{
Ricardo Ribeiro ${ }^{1}$, Eulália Costa ${ }^{1}$, Rui Bártolo ${ }^{1}$, Fernanda Fontes ${ }^{1}$, Fernando \\ Rodrigues ${ }^{1}$ \\ ${ }^{1}$ Department of Clinical Pathology, Centro Hospitalar e Universitário de Coimbra, \\ Coimbra, Portugal
}

Current clinical and laboratory guidelines suggest the use of non-ceruloplasmin bound copper (free copper) for the diagnosis and follow-up of patients with conditions associated with copper metabolism deregulation. However, formulas in use often result in inaccurate negative values, thus we sought to develop an equation adapted to our population in order to add analytical and clinical value to the test.

In the last 5 years, 1130 subjects with concomitant determination of total serum copper and ceruloplasmin at our institution were split into a training set (TS) ( $n=753$, after removal of participants with Wilson disease (WD) and abnormal copper levels) and validation set (VS) ( $n=396)$. Data from the training set was used to derive an equation through multivariate linear regression, which included total copper as dependent and ceruloplasmin, age and gender as independent variables, and considering its median serum copper value. This analysis yielded a good level of prediction $\left(r=0.802, r^{2}=0.643\right)$. The median $(95 \% \mathrm{Cl})$ for the adjusted copper in the TS was $39.0(29.5-53.1) \mu \mathrm{mol} / \mathrm{L}$.

Regression analyses in the VS after calculation using the equation for adjusted copper, demonstrated it was well related with measured total copper $\left(r=0.994, r^{2}=0.987\right)$, and ceruloplasmin $\left(r=0.818, r^{2}=0.669\right)$, and calculated free copper index $(r=0.812$, $\left.r^{2}=0.659\right)$ and copper-to-ceruloplasmin ratio $\left(r=0.416, r^{2}=0.173\right)$. Moreover, we used the lower limit of the $95 \% \mathrm{Cl}$ as cutoff to analyse its clinical value for detecting WD in the VS. The sensitivity and specificity of the adjusted copper for detecting WD was $68.4 \%$ (43.5-87.4) and $94.4 \%$ (91.6-96.5), respectively.

The use of adjusted copper derived from an equation from the Laboratory's own values can add information to the interpretation of copper levels while adjusting to the ceruloplasmin level. Further studies on larger samples with a wider concentration range may clarify the clinical utility of this instrument. 
P05

EVALUATION OF VITEK MS MYCOBACTERIUM/NOCARDIA EXTRACTION KIT AND VITEK MS V3 FOR NOCARDIA SPECIES IDENTIFICATION

\author{
Angélica Ramos ${ }^{1,3}$, Teresa Carvalho ${ }^{1}$, Masahiro Toyokawa ${ }^{2}$, Manuela Ribeiro ${ }^{1}$ \\ 1 Clinical Pathology Department of São João Hospital Center, Porto, Portugal; 2 Fukushima \\ Medical University, Fukushima, Japan; 3 EPIUnit - Institute of Public Health, University of Porto, \\ Porto, Portugal.
}

Introduction: In a laboratory where gene sequencing is not available, Nocardia identification to the species level can be difficult. The objective of this study was to evaluate the performance of VITEK ${ }^{\circledR}$ MS Mycobacterium/Nocardia extraction kit and VITEK $^{\circledR}$ MS version 3.0 database for Nocardia species identification.

Material and Methods: As a reference method, 59 clinical isolates were identified by $16 \mathrm{~S}$ rRNA gene sequencing. Nocardia were cultured on Middlebrook Agar 7H11 for $48 \mathrm{~h}$ and then isolates were prepared for VITEK MS V3 analyses through two different extraction methods. In the 1st method, each isolate was applied directly on the slide with $70 \%$ formic acid. In the 2 nd method, each isolated were prepared using a Mycobacterium/Nocardia extraction kit according to the manufacturer's instructions. Four spots per isolate were tested for both methods of sample preparation.

Results: With a single spot tested, $49.2 \%$ (29/59) and $84.7 \%$ (50/59) of Nocardia isolates were correctly identified at species or complex level through the 1st and the 2nd methods, respectively. With the second, third and four respotted, these values increase respectively to $67.8 \%(40 / 59), 84.7 \%(50 / 59)$ and $93.2 \%(55 / 59)$ with the 1 st method, and the use of the 2 nd method allowed to achieved values of $89.8 \%(53 / 59)$, $94.9 \%(56 / 59)$ and $94.9 \%(56 / 59)$, respectively. With 2 nd method, no increment was found when the fourth spot was tested.

Conclusions: The VITEK MS Mycobacterium/Nocardia extraction kit together with VITEK MS V3 may be useful for the routine identification of Nocardia in clinical practice. But, we also found that when three spots per isolate from $70 \%$ acid formic extraction were tested, the sensitivity of VITEK MS V3 is the same that was found when a single spot per isolate from VITEK MS extraction kit was performed. This data can be important for those laboratories that for various reasons (cost, turnaround time or expiration date) want to avoid acquiring the commercial kit. Despite these promising results, we concluded that the VITEK MS database needs to be improved, as the subspecies within the $N$. nova complex, the most frequent among our clinical isolates, could not yet be differentiated with this version. 
P06

PRE-ANALYTICAL PHASE: ARE THE CLINICIANS TELLING US EVERYTHING?

Marco P. Barros Pinto, Guilhermina Gaião Marques

Hospital de Santa Maria - Centro Hospitalar Universitário Lisboa Norte, EPE

Introduction: clinical and therapeutic information play a key role in the interpretation and validation of results in laboratory, avoiding the repetition of tests and allowing the association of small messages with validated results, such as the information of possible interferences of a drug on an analytical result.

Yao et al (2016) refer that antibacterial, psychotropic agents and contrast media are the drugs more likely to cause analytical interferences. Therefore, clinicians should pay attention when making a diagnosis or when defining a patient's status based on assay results in patients receiving these classes of drugs. For instance, the clinical laboratory tests most susceptible to a possible interference by the cephalosporins are urine glucose and ketone tests.

The aim of this work is to characterize the clinical and/or therapeutic information provided by clinicians, so as to establish an awareness program if needed.

Statistical analysis: We have analyzed a total of 1000 analytical requisitions, randomly selected, from hematology, internal medicine, pediatrics, infectiology and endocrinology departments (200 requisitions for each department), considering an ideal situation to be the existence of clinical and therapeutic information in all the requisitions.

Only $14.7 \%$ of the requisitions presented both informations, $52.1 \%$ had only clinical information, $33 \%$ did not have any information and $0.2 \%$ had only therapeutic information. When it comes to individual departments, the service that provided both informations more often ( $32.5 \%$ of their requests) was infectiology. On the other extreme, concerning the medicine department, $56.5 \%$ of the requisitions did not present any informations.

Clinical information was provided in $66.8 \%$ of requisitions $(84.5 \%, 75 \%, 70 \%, 61 \%$ of requisitions of infectiology, pediatrics, hematology and endocrinology, respectively).

Discussion: Clinical or therapeutic information provided by clinicians is clearly insufficient.

The results highlight the need to develop awareness programs in order to promote the importance of providing clinical and therapeutic information, at least in the departments studied.

We also agree that medicines leaflets should include information about the possible interferences that the drugs in question may have in laboratory analytical determinations. 
P07

THE INFLUENCE OF IRON STATUS ON THE HBA2 WINDOW IN HPLC - HEREDITARY HEMOCHROMATOSIS PATIENTS UNDER INTENSIVE TREATMENT AS A MODEL

João Pedro Barreto1, Sandra Martins ${ }^{2,3}$, Graça Melo ${ }^{4}$, Graça Porto ${ }^{4,5,6,7}$, Maria José Teles ${ }^{2,5,8}$

${ }^{1}$ Diagnostic Laboratory Department, Portuguese Oncology Institute, Porto ${ }^{2}$ Clinical Pathology Department, Sao Joao Hospital and University Centre ${ }^{3}$ EpiUnit, Public Health Institute, University of Porto

${ }^{4}$ Clinical Hematology Department, Porto Hospital and University Center

5 Institute for Research and Innovation in Health (I3S)

${ }^{6}$ Institute for Molecular and Cell Biology, University of Porto

${ }^{7}$ Abel Salazar Institute of Biomedical Sciences, University of Porto

${ }^{8}$ Faculty of Medicine, University of Porto

Hemoglobin A2 ( $\mathrm{HbA} 2$, a $\alpha 2 \delta 2$ heterodimer) represents 2,5-3,5\% of the total hemoglobin in an adult. It is particularly important in the diagnosis of $\beta$-thalassemia, in which a higher value of $\mathrm{HbA} 2$ is detectable by High-Pressure Liquid Chromatography (HPLC). A common confounding factor in this context is the presence of iron deficiency, since it will lower the value of $\mathrm{HbA} 2$ and can result in a falsely normal value in $\beta$ thalassemia patients.

Hereditary Hemochromatosis $(\mathrm{HH})$ is a rare disease that leads to an iron overload and the recommended treatment is an intensive regime of phlebotomies, so that the iron in excess is mobilized until reaching iron deficiency (Transferrin Saturation $<20 \%$ or Ferritin $<50 \mathrm{ng} / \mathrm{mL}$ ). Therefore, $\mathrm{HH}$ patients under intensive treatment offer a good model to study the relationship between iron stores and the value of $\mathrm{HbA} 2$.

We compared the values of Ferritin/Transferrin saturation and $\mathrm{HbA} 2$ in 9 patients diagnosed with $\mathrm{HH}$, in the beginning and at the end of an intensive phlebotomy treatment.

In all the patients there was a significant drop in ferritin and/or transferrin saturation values, as expected. The percent values of $\mathrm{HbA} 2$ dropped in 8 of these patients, with a mean drop of $0,225 \%$ (minimum: $0,1 \%$, maximum: $0,3 \%$ ). There was a patient in which the $\mathrm{HbA} 2$ percent remained stable. This is a patient under study for a chronic inflammatory disease, with a high value of C-reactive protein, that can be a potential biologic confounding factor.

These results highlight the need to correct iron deficiency before studying any patient for $\beta$-thalassemia, in order to avoid a false lower level of HbA2. 
P08

ADULT MALARIA INFECTION AS CAUSE OF SEPSIS

Catarina Machado Ferreira, Cláudia Reynolds, Joana Sevilha, Paula Costa, Marvin Oliveira, Maria Calle, Marília Dias

Centro Hospitalar Tâmega e Sousa

Introduction: Malaria, caused by a protozoa of the genus Plasmodium, is the most important protozoan disease in the world and a global public health issue nowadays. Severe cases can complicate with organ dysfunction and sepsis. The following clinical case emphasizes the role of the laboratory diagnosis on early treatment, therapy follow-up and survival rates in this disease.

Description: A 42-year-old male, living in Angola for two years, presented to our Emergency Department (ED) with headache, myalgias and fever for the past 5 days. Past medical history included 2 episodes of malaria and an episode of septic arthritis from Chikungunya virus infection in the last two years. Patient had returned 12 days ago from Angola. At physical examination patient showed hypotension, hypoglycemia, fever and signs of respiratory distress. His abnormal blood laboratory findings on admission were as follows: normocytic normochromic anemia(11,9 $\mathrm{g} / \mathrm{dL})$, thrombocytopenia( $\left.47 \times 10^{3} / \mathrm{uL}\right)$, hyponatremia and elevated C-reactive protein $(225$ $\mathrm{mg} / \mathrm{L})$. Blood smears were positive for Plasmodium infection with a $5 \%$ parasitaemia and reticulocyte count was reported at $0,8 \%$. These findings were confirmed by in vitro immunochromatographic assay BinaxNOW $\AA$, that showed positive for P.Falciparum or mixed infection. Due to the presence of severe malaria criteria compatible with sepsis, was transferred to the medical intensive care unit for further care. SOFA and APACHE II initial scores were both 5 with $20 \%$ and $8 \%$ of mortality rate, respectively. The patient was treated with intravenous Quinine and Doxycycline and monitored with consecutive blood smears that revealed progressive decrease of parasitaemia. The patient responded well to treatment, and 5 days later left the hospital against medical advice. At home, under supervision of his Family Physician he completed oral therapy successfully.

Discussion: Severe malaria is a medical emergency and should be diagnosed and treated early, highlighting the laboratory diagnosis for this purpose. Its prognosis is invariably related to early initiation of treatment and monitoring of parasitemia, where Laboratory Hematology plays a fundamental role. Malaria can cause millions of deaths per year. Awareness should be raised and laboratory support strengthen in the management of these disease. 
P09

APPLICATION OF THE SIX SIGMA METHODOLOGY IN THE EVALUATION OF THE RESULTS IN CELL BLOOD COUNT EQAS PROGRAM 2015-2017 (PNAEQ)

\author{
Ana Raquel Ovelheiro ${ }^{1,2}$, Armandina Miranda ${ }^{1}$, Helena Correia ${ }^{1}$, Ana Cardoso ${ }^{1}$, Ana \\ Faria $^{1}$, José Gomes Requeijo ${ }^{2}$ \\ ${ }^{1}$ Programa Nacional de Avaliação Externa da Qualidade (PNAEQ), Unidade de Avaliação \\ Externa da Qualidade, Departamento de Epidemiologia, Instituto Nacional de Saúde Doutor \\ Ricardo Jorge, Lisboa, Portugal \\ ${ }^{2}$ Departamento de Engenharia Mecânica e Industrial, Faculdade de Ciências e Tecnologias, \\ Universidade Nova de Lisboa, Lisboa, Portugal
}

Introduction and objective: The haemogram is one of the most frequently requested laboratory tests, in hospital and ambulatory. Therefore, given its importance in the clinical context, an evaluation was performed on the results of the clinical laboratories participating in PNAEQ's EQA Cell Blood program from 2015 to 2017. The main objective of this work was to improve the Sigma quality level of the clinical laboratories and reduce the variability of their results.

Methods: For the calculation of the Six Sigma metric, the inaccuracy (bias) associated to the result obtained by each laboratory for different parameters of each sample was determined and the outlier's treatment was performed. In the first approach, evaluation per sample, the Normality of each sample results was studied by applying the Kolmogorov-Smirnov test. The Box-Cox transformation was applied whenever necessary. Regarding haemoglobin parameter, a second approach, namely the linear regression was applied. This model allows establishing a comparison between the laboratories' results and the consensus value, obtained by the average of the participating laboratories. The Sigma quality level for both approaches was obtained considering the desirable quality specification based on the biological variation.

Results and discussion: After the statistical analysis of the results, the mean Sigma quality level in the sample approach was 1.95, 1.71, 2.22 and 1.57 for the parameter haemoglobin, platelets, leukocytes and erythrocytes, respectively. The mean Sigma quality level obtained in the laboratory approach for the parameter haemoglobin was 2.64. Both approaches demonstrated a need to improve the analytical process performance. Therefore, in brainstorming meetings with the participants were identified, in the Analyze phase, some potential causes for the low performance. Posteriorly, in the Improve phase, improvement actions were elaborated and implemented. Through the pilot test, it was possible to verify improvements in the analytical performance of the laboratories, obtaining a Sigma quality level of 2.62, 2.58, 2.27 and 1.87 for the parameter haemoglobin, platelets, leukocytes and erythrocytes, respectively.

Conclusions: Continuous monitoring of the processes will be carried out, aiming to ensure that the implemented improvements continue to be practiced. 
P10

\section{CAN SERUM FREE LIGHT CHAIN RATIO SUBSTITUTE URINE BENCE JONES DETECTION? - A RETROSPECTIVE STUDY IN CENTRO HOSPITALAR DE LISBOA OCIDENTAL}

$\underline{\text { Rita Paulino }}{ }^{1}$ Alexandra Mendes ${ }^{2}$, Ana Isabel Matos, Ana Torrinha, Raquel Serra, Isabel Baptista Fernandes, João Faro Viana

Serviço de Patologia Clínica, Centro Hospitalar de Lisboa Ocidental

1,2 These authors contributed equally to this work

Introduction: Monoclonal gammopathies are a group of disorders characterized by the proliferation of a single clone of plasma cells that produce a monoclonal protein. This protein can be detected by electrophoresis, immunofixation or by measurement of serum free light chain (sFLC). Bence Jones (BJ) proteinuria refers to monoclonal free light chains in urine.

Objective: To evaluate if sFLC ratio ( $\mathrm{sFLCr}$ ) can substitute urine $\mathrm{BJ}(\mathrm{uBJ})$ detection.

Material and Methods: We performed a retrospective study with data collected between 2008 and 2019 from our Hospital. With a total of 1329 patients with monoclonal gammopathies (Multiple Myeloma, Monoclonal Gammopathy of Undetermined Significance, Amyloidosis, etc.), 754 were selected on the basis of having both sFLCr and uBJ (370 men and 384 women, with ages between 32 and 98 years old, median of 72 years old). The sFLC assay was performed by nephelometry (Atellica Neph 630 тм - Siemens) and the ratio calculated, and uBJ were identified by immunofixation (Hydrasys ${ }^{\mathrm{T} M}$ system - Sebia), after urine concentration (10x). We assessed the degree of agreement using Cohen's kappa (k).

Results and discussion: From the 754 patients selected 394 (52\%) had BJ proteinuria (233 BJ kappa, 158 BJ lambda, 3 with both) and 360 (48\%) did not.

There is a moderate concordance between sFLC ratio and urine BJ (\% of agreement 70.42; Cohen's k=0.41).

\begin{tabular}{|l|l|l|}
\hline & Positive BJ & Negative BJ \\
\hline Altered sFLC ratio & 275 & 104 \\
\hline Normal sFLC ratio & 119 & 256 \\
\hline
\end{tabular}


Of the positive BJ with normal sFLC ratio, 99 (83\%) had an increased sFLC.

\begin{tabular}{|l|l|l|l|}
\hline & \multicolumn{3}{|l|}{ Positive BJ } \\
\hline & Total & Kappa & Lambda \\
\hline Both sFLC elevated & $51^{*}$ & 32 & 20 \\
\hline sFLC kappa elevated & 14 & 12 & 2 \\
\hline sFLC lambda elevated & 34 & 19 & 15 \\
\hline Normal range sFLC & 20 & 1 & 19 \\
\hline Total & 119 & 64 & 56 \\
\hline
\end{tabular}

*One of the samples had both BJK and BJL

The altered sFLCr without BJ proteinuria could be due to normal kidney capacity to reabsorb the BJ proteins or to sampling problems, especially those associated to the urine collection.

The cases with a normal sFLCr but with a uBJ detected could be justified by renal conditions (inability to reabsorb BJ proteins) or when a polyclonal production of FLC exists and the monoclonal FLC is not sufficient to alter the ratio.

Conclusion: As $15 \%$ of our cases with a positive $\mathrm{UBJ}$ had a normal sFLCr, we can conclude that the SFLCr cannot substitute the urine detection of BJ protein. 
P11

COMPARATIVE STUDY OF ZINC DETERMINATION

\author{
$\underline{\text { Ana Cunha Lima }}{ }^{1}$, Ana Aguiar $^{1}$, Mariana Silva $^{1}$, Eugénia Lobo $^{1}$, Miguel Pinto da Costa $^{1}$ \\ ${ }^{1}$ Serviço de Patologia Clínica do Centro Hospitalar Entre Douro e Vouga (CHEDV) \\ Diretora de Serviço Patologia Clínica do CHEDV - Dr 므 Ana Cristina Silva
}

Zinc is a mineral that is distributed throughout the body in small concentrations. Its deficiency is associated with serious pathological conditions that arise mainly due to malnutrition, changes in gastrointestinal absorption or increase in urinary excretion. The malabsorption and consequent Zinc deficiency were demonstrated in obese patients undergoing bariatric surgery. In these patients, many factors contribute to Zinc deficiency, these include malabsorption caused by the bypass of the duodenum and proximal jejunum (preferential location for Zinc absorption), intolerance to Zinc rich foods (red meats) and reduced gastric hydrochloric acid production, essential for the absorption of this mineral.

In 2017, our hospital received the status of a Center for Surgical Treatment of Obesity, directly increasing the number of bariatric surgeries and, consequently, the requests for Zinc determination, which was initially requested to an outside laboratory.

Objectives: Access the possibility of determination of Zinc concentration internally, instead of referring to an external entity.

Materials and Methods: 42 serum samples were analysed in parallel over a period of 2 months: the reagent already in use by the outside laboratory, named Randox; and one named BioSystem, which our Laboratory wished to adopt. Both are spectrophotometric assays. For result assessment, Laboratory Information System Appolo ${ }^{\circledR}$ was used as the main application, while Microsoft Excel $^{\circledR}$ and SPSS ${ }^{\circledR}$ were used for statistical treatment.

Data was analysed using linear regression and Pearson's correlation coefficient.

Results: About $95 \%$ of Zinc dosing requests are pre and postoperative of Bariatric Surgery, and have increased considerably in number in recent years ( $n=606$ in 2013; $\mathrm{n}=1564$ in 2018).

Considering the 42 analysed samples, a Pearson correlation of 0.784 and linear regression $(y=11.0242+0.6759 x)$ was obtained.

Conclusion: The results with the different reagents, Randox and BioSystem, are comparable, showing a significant strong positive correlation $(\rho=0.784)$.

Based on the number of requests for Zinc determination, and the strong correlation of both methods, the internalisation of Zinc determination in our hospital was considered viable, providing cost reduction and better response times. 
P12

\section{ROLE OF FLOW CYTOMETRY IN A SOLID TUMOR}

Carlos Martins ${ }^{1}$; Joana Sevilha ${ }^{2}$, Sara Braga ${ }^{3}$; Cátia Rodrigues ${ }^{3}$; Cátia Mendes ${ }^{3}$; Carlos Palmeira ; M. Emília de Sousa ${ }^{3}$; Inês Godinho ${ }^{3}$; Ana Pires ${ }^{3}$; Carla Azevedo ${ }^{3}$; Ângelo Martins $^{4}$; Gabriela Martins ${ }^{3}$

${ }^{1}$ Serviço de Patologia Clínica, Centro Hospitalar Vila Nova de Gaia/Espinho, EPE

${ }^{2}$ Serviço de Patologia Clínica, Centro Hospitalar do Tâmega e Sousa, EPE

${ }^{3}$ Serviço de Imunologia, Instituto Português de Oncologia do Porto Francisco Gentil, EPE

${ }^{4}$ Serviço de Onco-Hematologia, Instituto Português de Oncologia do Porto Francisco Gentil, EPE

Introduction: Pediatric cancer is a relatively rare and heterogeneous group of hematological and non-hematological malignancies which require multiple procedures for its diagnostic screening and classification. In this case report we explored the potential application of flow cytometry (FC) in a solid tumor.

Clinical case: A 13-year-old male was admitted in our hospital with bilateral, enlarged, non-adherent, mobile, cervical lymph nodes, hard right inguinal node and a hard mass in the perineum area. MRI and Inguinal ultrasound showed a voluminous mass, suggestive of a prostate sarcoma. Histological, genetic and immunophenotypic studies were performed through the biopsy. For this purpose, a set of antibodies panel was used as well as the proliferative index evaluation through the study of DNA content. The immunophenotypic characterization revealed the presence of $24 \%$ of pathological population with phenotype CD9+ CD34- CD45- CD56+/++ cCD68- CD81+ CD90- BerEP+ $\operatorname{dim}$ cytokeratin, DNA index of 2.39, hypertetraploid population and a high proliferative index (7.13\% of cells at S phase). The results were compatible with a nonhematological disorder. Genetic studies revealed that $89 \%$ of cells had a FOXO1 (FKHR) (13q14) gene rearrangement. This result was consistent with alveolar rhabdomyosarcoma.

Discussion: Rhabdomyosarcoma (RMS) is the most common type of soft tissue sarcoma in children. The bladder/prostate region is a frequent site of occurrence. RMS is a highly malignant tumour with varying clinical behaviour and histopathological presentation. Despite cancer is the leading cause of disease-related death in children in most countries, individual outcomes largely depend on fast tumor diagnosis for appropriate therapy selection. The flow cytometry provides a more rapid immunophenotyping than conventional immunohistochemical staining. Although flow cytometry does not detect a specific immunophenotype for Rhabdomyosarcoma, it allowed in this case to identify rapidly as a nonhematopoietic neoplasm which was important for therapeutic decision making. 
P13

LACTATE DEHYDROGENASE STABILITY IN SERUM AT DIFFERENT TEMPERATURES AND STORAGE TIMES

Mónica Peixe, Ricardo Fernandes, Isabel Galvão

Serviço de Patologia Clínica - Hospital Beatriz Ângelo - Synlab Portugal

Introduction: Information on the stability of lactate dehydrogenase (LDH) during storage of serum samples lacks consensus.

There are conflicting data regarding samples kept at room temperature (RT) and at $4^{\circ} \mathrm{C}$, with reported stabilities from 0 to 6 days or more, in both storage conditions. Previous studies present different designs and criteria for data analysis. Most of them use low number of samples and include only healthy individuals. These factors may be the cause of such discrepancies.

Understanding how LDH concentration changes over time during storage is essential for proper clinical interpretation of results in case of reanalysis or add-on requests, very common in emergency departments.

Objective: Determine the effects of storage time and temperature on LDH concentration and evaluate sample suitability for reanalysis and add-on requests.

Methods: LDH was measured in serum samples from 74 patients (range: 110 to 833 $\mathrm{U} / \mathrm{L}$ ) using Roche Cobas ${ }^{\circledR} 6000$. After initial measurement samples were aliquoted into fifteen tubes and stored at three different temperatures: frozen $\left(-80^{\circ} \mathrm{C}\right)$, refrigerated $\left(4^{\circ} \mathrm{C}\right)$ and $\mathrm{RT}\left(24^{\circ} \mathrm{C}\right)$. LDH was then measured for five consecutive days.

Differences between LDH concentration at baseline and concentrations measured at subsequent time points, following different storage conditions, were calculated as Mean Percentage Deviation (MD\%).

Evaluation was against the Total Change Limit (TCL), described previously by Oddoze et al. for stability studies, which represents the allowable clinical percentage change. The TCL was calculated using the within-subject biological variation (Carobene et al.) and analytical imprecision (cumulative CV\% over a 6-month period). ( $T C L= \pm 6,25 \%$ )

Results: LDH showed no significant change for all storage conditions up to $48 \mathrm{~h}$. However, on the third day, MD\% exceeded the TCL for samples stored at $4^{\circ} \mathrm{C}$ (MD\%=$8,39 \%)$. Percentage change of samples kept at $-80^{\circ} \mathrm{C}$ and at RT were within the TCL over the five-day period.

Conclusions: LDH analysis or reanalysis can be performed within $48 \mathrm{~h}$ of specimen collection if serum is kept at $4^{\circ} \mathrm{C}$ and for at least 5 days if stored at RT or at $-80^{\circ} \mathrm{C}$.

Knowing the stability of analytes is vital for adequate interpretation of results from add-on requests and retesting. 
P14

MYCOTIC ANEURISM OF ABDOMINAL AORTA BY SALMONELLA TIPHYMURIUM: A CASE REPORT

Teresa Reis ${ }^{1}$; Anália Carmo ${ }^{1}$; Fernando Rodrigues ${ }^{1}$; Catarina Chaves ${ }^{1}$
${ }^{1}$ Clinical Pathology Department, Coimbra University Hospital Center (CHUC), Coimbra, Portugal

Introduction: Mycotic aneurysm (MA) is a rare, life-threatening pathologic entity that results from bacterial or fungal infection of the arterial wall. Salmonella spp. (SPP) is one of the most common strains associated to this illness. We present a case-report of a MA of the abdominal aorta in a patient infected by Salmonella tiphymurium (ST).

Case Report: An eighty-year-old caucasian male patient under clinical investigation due a suspicion of myelodysplastic syndrome (MDS) was admitted the emergency room due to the worsening of asthenia and anorexia. Unless the suspicion of MDS, his past medical history was unremarkable. Physical examination evidenced a mucocutaneous pallor, without lymphadenopathy or organomegaly. Laboratory tests showed hemoglobin $9.3 \mathrm{~g} / \mathrm{dL}, \mathrm{WBC} 7.7 \mathrm{~K} / \mathrm{uL}$, platelet count $422 \mathrm{~K} / \mathrm{uL}$, calcium 8.7 $\mathrm{mg} / \mathrm{dL}$ C-reactive protein $74.93 \mathrm{mg} / \mathrm{L}$. The patient was transferred to a medical ward to continue investigation. Blood was collected for microbiology. On the third day, due to a reduction in hemoglobin $(7.3 \mathrm{~g} / \mathrm{dl})$ and to the development of fever, the patient performed a blood transfusion and started piperacillin-tazobactam. On the eighth day, a ST was identified in blood cultures, and antibiotic was changed to ciprofloxacin according the antibiogram (ATB) results. Due to the deterioration of clinical condition he performed a chest-abdomen-pelvis CT that evidenced the presence of an aneurysm of the abdominal aorta. The patient underwent surgery to perform the excision of the aneurysm. An aortic fragment was sent to laboratory and a smear was performed. The biopsy was cultured in the appropriate media and incubated in aerobic and anaerobic atmosphere. A ST was identified by mass spectrometry and serologic tests confirming the existence of the MA. ATB was performed by automatic equipment. Patient maintained antibiotic therapy and achieved a full recovery.

Discussion: Salmonella spp. has a strong affinity for large blood vessels. In older patients with atherosclerotic vascular wall, the adhesion of SPP may induce the vascular wall rupture with subsequent haemorrhage. In $70 \%$ of cases, fever and leukocytosis are usually the first findings making difficult the diagnosis. The prompt identification of SPP in microbiologic culture is crucial to the diagnosis and to the beginning of the appropriate treatment. 
P15

PNAEQ PERFORMANCE ASSESSMENT OF VIRUS INFLUENZA A E B PCR DETECTION (2011-2018)

Helena Correia ${ }^{1}$, Ana Cardoso ${ }^{1}$, Edna Pereira ${ }^{1}$, Catarina Ventura ${ }^{1}$, Sílvia Viegas ${ }^{1}$, Neuza Gomes $^{1}$, Marina Cordeiro ${ }^{1}$, Paula Cristóvão ${ }^{2}$; Raquel Guiomar ${ }^{2}$; Ana Faria ${ }^{1}$

${ }^{1}$ Instituto Nacional de Saúde Dr. Ricardo Jorge - Departamento de Epidemiologia - Unidade de Avaliação Externa da Qualidade, Avenida Padre Cruz, 1649-016 Lisboa, Portugal. pnaeq@insa.min-saude.pt

2 Instituto Nacional de Saúde Dr. Ricardo Jorge - Departamento de Doenças Infecciosas Laboratório Nacional de Referência para o Vírus da Gripe e Outros Vírus Respiratórios

Introduction: Influenza viruses are responsible for respiratory infections that occur seasonally, usually in the winter, or as periodic and unpredictable pandemics.

In 2011 PNAEQ implemented, in collaboration with the National Reference Laboratory for Influenza and Other Respiratory Viruses of the National Institute of Health Dr. Ricardo Jorge, the External Quality Assessment Program for the influenza A and B viruses, nucleic acid detection.

Objective:_Performance evaluation of the laboratories participating in the PNAEQ in the detection and identification of type and subtype of influenza viruses, using PCR methodology, in the period 2011-2018.

Material e Methods:_Fifty-six control samples were analyzed by the participating laboratories, between 2011-2018. Of the samples submitted, 30 presented influenza $A, 1$ sample subtype $A(H 1 N 1), 15$ subtype $A(H 1 N 1) p d m 09$ and 14 subtype $A(H 3 N 2)$. 16 samples were type $B$ and 10 were negative.

The results were statistically evaluated with reference to information from the Expert Laboratory (National Reference Laboratory for Influenza Virus and Other Respiratory Viruses).

Results: In the period under study, a percentage of correct results was obtained of 98.6\% for influenza A, $95.2 \%$ for influenza B and $96.5 \%$ for negative samples.

Of the results analyzed, 10 false positive results $(1.5 \%)$ and 9 false negative results $(1.3 \%)$ were obtained.

Discussion/conclusion: The results obtained by the participating laboratories were considered good, with correct results for more than $95 \%$ of the samples tested. Participation in external quality assessment programs allows laboratories to evaluate the methodologies and practices implemented, as well as correcting systematic errors, thus ensuring comparability and harmonization of the results of laboratories responsible for the diagnosis of influenza virus. 
P16

PULMONARY ADENOCARCINOMA COEXISTING WITH ACUTE MYELOID LEUKAEMIA - A CLINICAL CASE

Hugo Loureiro ${ }^{1}$, Sara Braga ${ }^{2}$, Joana Sevilha ${ }^{3}$,Carlos Martins ${ }^{4}$, Teresa Sousa ${ }^{5}$, Carlos Mendes ${ }^{6}$

${ }^{1}$ Serviço de Patologia Clínica do Centro Hospitalar Universitário São João

2 Serviço de Hematologia Laboratorial do Instituto Português de Oncologia do Porto (IPO)

${ }^{3}$ Serviço de Patologia Clínica do Centro Hospitalar do Tâmega e Sousa

${ }^{4}$ Serviço de Patologia Clínica do Centro Hospitalar de Vila Nova de Gaia e Espinho

${ }^{5}$ Serviço de Hematologia Laboratorial do Instituto Português de Oncologia do Porto (IPO)

${ }^{6}$ Serviço de Hematologia Laboratorial do Instituto Português de Oncologia do Porto (IPO)

Introduction: Multiple Primary Cancers (MPCs) consist of more than one primary tumour diagnosed in the same patient simultaneously, each tumour anatomically distant from the other. The possibility that one represents metastasis of the other must be excluded. MPCs are rare, with bad prognosis and mainly involve gastrointestinal and breast cancers, as well as haematological malignancies.

Secondary haematological malignancies represent a rare but serious complication of oncological treatment. Studies show that after chemotherapy the risk of leukaemia arises about 2 years after treatment with alkylate agents.

Case Report: We present a 75 year-old male patient, former smoker, with a history of exposure to industrial varnishes, followed in this hospital for Lung Adenocarcinoma in the superior segment of the inferior lobe of the left lung diagnosed on $11 / 2018$. On $04 / 02 / 2019$ he underwent left inferior lobectomy and mediastinal lymphadenectomy. On 10/02 he was admitted for respiratory infection. On the analytical study requested there were changes on the blood count: an abnormal scattergram, macrocytic anaemia and neutropenia de novo and a worsening of a pre-existing thrombocytopenia. The blood smear revealed the presence of circulating blast cells, suggesting a diagnosis of Acute Leukaemia.

Further studies were requested, including bone marrow biopsy and aspirate, immunophenotyping analysis and cytogenetic study. The patient had about $50 \%$ of blast cells, with a myeloid immunophenotype and an arrest in maturation, along with diserythropoiesis. Cytogenetics showed a high percentage of metaphases with $Y$ deletion. All these findings were compatible with a diagnosis of Acute Myeloid Leukaemia (AML).

Discussion: The evidence of a pre-existing thrombocytopenia and the absence of previous chemotherapy, leads us to believe that this patient had a MPC, with synchronous Lung Adenocarcinoma and AML. There was no evidence of a cause-effect relation between the two malignancies. This case shows the importance of the Clinical Pathologist in detecting the changes that rose the suspicion of AML before the existence of clinical signs or symptoms consequently leading to the investigation of a condition that changed the management of the patient, altering his prognosis and requiring a more challenging therapeutic control. 
P17

PSEUDO-PELGER-HUET ANOMALY IN A BONE MARROW TRANSPLANT PATIENT MYELODYSPLASTIC SYNDROME OR IATROGENIC?

\author{
Ana Torgal, Sara Braga, João Pedro Barreto, Carlos Mendes, Teresa Sousa
}

Instituto Português de Oncologia do Porto Francisco Gentil, EPE - Serviço de Hematologia Laboratorial

Introduction: Pseudo-Pelger-Huët anomaly (PPHA) may be a result of clonal myeloid malignancy or secondary to a variety of causes, including infections and drugs. In transplant recipients it is often attributed to immunosuppressive drugs.

Case report:_A 36-year-old woman with history of nodular sclerosis Hodgkin lymphoma developed PPHA following bone marrow transplant. 4,5 months after transplantation the patient presented with anemia (hemoglobin $(\mathrm{Hb}) 11,2 \mathrm{~g} / \mathrm{dl}$ ) and normal leucocyte count (WBC). Examination of the blood smear (BS) revealed some dysplastic neutrophils with nuclear hyposegmentation and abnormalities of chromatin condensation. The patient was on tacrolimus, mycophenolate mofetil (MMF) and valganciclovir, among other drugs, with no evidence of acute illness or infection.

Blood cell count (including WBC) continued to decrease and 5 weeks later she presented $\mathrm{Hb} 10,6 \mathrm{~g} / \mathrm{dl}$; WBC $3.0 \times 10^{9} / \mathrm{L}$; neutrophil $0.72 \times 10^{9} / \mathrm{L}$; platelets $195 \times 10^{9} / \mathrm{L}$, with the majority of neutrophils demonstrating PPHA. Tacrolimus and valganciclovir were discontinued.

Bone marrow aspirate (BMA) subsequently performed was hypocellular, with abnormal clumping of nuclear chromatin at all phases of myeloid development and paucity of segmented neutrophils. Megakaryocytes and erythroblasts presented mild dysplastic changes. Cytogenetic and imunophenotypic studies were normal. Considering the clinical-laboratorial context, the patient remained under close surveillance. Neutropenia resolution, with an improvement on neutrophil morphology was only attained 3 months after tacrolimus was suspended.

Discussion: Tacrolimus and MMF (either as single agents or in combination with other drugs, namely valganciclovir) have been considered causative agents of PPHA in transplanted patients. Morphologic neutrophil changes appear to precede the development of neutropenia, which seems to have a higher incidence in patients with PPHA. Thus, blood counts and BS should be monitored closely.

Transplanted patients are usually on polytherapy and it is impossible to discontinue all imunossupression, and assess the reversibility of PPHA. Nevertheless, iatrogenic PPHA should be kept in mind as a differential diagnosis with myelodysplastic syndrome or other myeloid malignancies, potentially preventing unnecessary interventions. 
P18

BRAIN TUMOR? A NOCARDIA ABCESSUS BRAIN ABSCESS CLINICAL CASE

João Gonçalo Frade ${ }^{1,2}$, Teresa Reis ${ }^{2}$, Susana Alarico ${ }^{3}$, Nuno Empadinhas ${ }^{3}$, Fernando Rodrigues ${ }^{2}$, Catarina Chaves $^{2}$

${ }^{1}$ School of Health Sciences, Polytechnic of Leiria, Leiria, Portugal

2 Clinical Pathology Department, Coimbra University Hospital Center (CHUC), Coimbra, Portugal

${ }^{3}$ Centre for Neuroscience and Cell Biology, University of Coimbra, Portugal

Introduction: Nocardiosis is an uncommon disease caused by aerobic gram-positive bacteria of the genus Nocardia that typically cause localized or systemic disease in both immunocompetent and immunocompromised subjects. Patients with invasive Nocardiosis are often seriously ill and published reports are scarce, particularly those addressing central nervous system (CNS). We present a case of a patient with a Nocardia abscessus (NA) brain abscess.

Case Report: A male, age 42, presented to the Emergency Room with mild frontal headache, incoherent speech and no history of trauma or complaints other than a lung mass, under investigation. Because a CT exam reported a "left postero-temporal hyperdensal lesion, with extensive perilesional edema" the hypothesis of left temporal cerebral tumor (of probable pulmonary origin) was introduced. Surgical approach to the lesion led to the excision of a temporal abscess and drainage of purulent contents. Gram staining revealed gram-positive thin branching filaments bacilli and, after incubation, growth occurred in chalky white colonies. Mass spectrometry identification of colonies revealed NA. Further identification was conducted by sequencing the 16S rRNA gene from the isolate (1398 bp). Nucleotide sequences were compared with those of representative Nocardia species in the NCBI Blast database. The phylogenetic analysis clustered the isolate with strains of NA (at 100\% identity, accession numbers MK311192, GenBank database). Medical treatment included meropenem, amikacin, trimethoprim/sulfamethoxazole and ceftriaxone according to available literature for treatment of nocardiosis, adapted according to disease progression. During the hospitalization the patient was also diagnosed with pulmonary aspergillosis and treated with voriconazole, with a successfully discharge 168 days latter without clinical sequels. The rarity of CNS Nocardiosis was supported by local epidemiological results from the past 10 years, where patients presented with pulmonary and cutaneous infections.

Discussion: The CNS is considered a rare extrapulmonary location for nocardiosis. As Nocardia remains a formidable pathogen in immunocompetent/immunocompromised subjects, early recognition and initiation of appropriate treatment can lead to successful outcomes. 
P19

Severe Malaria in an Intensive Care Unit: a case report

Sofia Novo, Nuno Braga, Paula Mota

Hospital Senhora da Oliveira, Guimarães, E.P.E.

Introduction: P. falciparum malaria is an important cause of morbidity and mortality worldwide. It is not endemic in Europe and reported cases are almost exclusively in travelers returning from malaria-endemic areas. Nonspecific clinic is frequently associated and definitive diagnosis goes through the demonstration of the parasite or its antigens by laboratory tests.

Case Report: A 54-year-old male patient presented with 2-day course of general malaise, asthenia, anorexia and polydipsia, since returning from Angola without complying antimalarial prophylaxis. Medical history: hypertension, dyslipidemia, type 2 diabetes mellitus, obesity, smoking, alcoholism and complicated malaria 5 years ago. Physical examination: disorientation, dehydration of the skin and mucous membranes, icteric sclerotic and signs of respiratory distress (SatO2 87\%). Laboratory test results showed thrombocytopenia $29 \times 10^{3} / \mu \mathrm{L}$, mild leukopenia, hyperbilirubinemia, lactate dehydrogenase level $517 \mathrm{U} / \mathrm{L}, \mathrm{C}$ reactive protein level 178 $\mathrm{mg} / \mathrm{L}$ and hyperlactacidemia. Microscopic slides of thick and thin May Grunwald Giemsa stained blood smears showed the presence of trophozoites and gametocytes morphologically compatible with P. falciparum with $25 \%$ parasitaemia. Rapid immunochromatographic diagnostic test (RDT) (BinaxNow ${ }^{\circledR}$, Alere) confirmed the presence of $P$.falciparum in the blood sample.

The pacient was treated with quinine, artemether-lumefantrine and doxycycline. After 48 hours, due to progressive worsening of the condition, he was admitted to the Intensive Care Unit for severe malaria complicated with Multiorganic Dysfunction Syndrome (respiratory, hematological, neurological and metabolic). Parasitaemia decreased with treatment, being negative on the 14th day. As intercurrences he presented ventilator-associated pneumonia and toxic hepatitis, being discharged at day 28 clinically and analytically improved.

Conclusion: Although Portugal ceased to be an endemic area around 1950, imported cases of malaria tend to increase due to the mobility of people in endemic areas. Prompt, accurate diagnosis is part of effective management of disease. All patients with suspected malaria should be treated on the basis of a confirmed diagnosis by microscopy examination, RDT or molecular technics of a blood sample.

\section{References}

Blood cells, a practical guide. Bain, Barbara J.(2015). John Wiley \& Sons Ltd.

www.uptodate.com. Acessed on-line on 30-01-2019

BMJ Publishing Group, Ltd 2018. Acessed on-line on 10-01-2019

WHO website, Accessed on-line on 12-2-2019

www.cdc.gov. Accessed on-line on 12-2-2019 
P20

FLOW CYTOMETRIC CELL PATTERN OF BRONCHOALVEOLAR LAVAGE IN PULMONARY DISEASES: SMOKERS VERSUS NON-SMOKERS

Mariana Feijó, Andreia Monteiro, Patrícia Amantegui, Cristiana Reis, Sara Correia, Maria Jesus Valente, Maria Conceição Faria

Universidade da Beira Interior

Over the years, flow cytometric analysis of bronchoalveolar lavage (BAL) has become an essential procedure in differential diagnosis of pulmonary diseases (PD). In healthy subjects, BAL's cell pattern is notoriously different between smokers and nonsmokers. The present study aims to understand if and how smoking habits influence the BAL's cell pattern of patients with PD. Between 2014 and 2018, our institution performed $285 \mathrm{BAL}$, which cell surface antigen expression of total lymphocytes, CD4 ${ }^{+}$ and $\mathrm{CD}^{+} \mathrm{T}$ cells and macrophages was characterized by flow cytometry. The study population includes 148 men and 137 woman, with age comprised between 17 and 87 years and mean age of 61 years, 179 non-smokers, 60 ex-smokers and 46 smokers. This population includes cases of pulmonary nodule (PN), pneumonia, idiopathic pulmonary fibrosis (IPF), sarcoidosis, bronchial asthma (BA) and chronic obstructive pulmonary disease $(C O P D)$. Statistically significant differences $(P<0.05)$ between the tested groups were obtained by Kruskal-Wallis test followed by Mann-Whitney test, utilizing IBM SPSS Statics (version 25) software. Across all study groups, smokers showed an increased macrophages' frequency in comparison to non-smokers, being statistically significant in pneumonia and BA. Relatively to CD4/CD8 ratio, no significant differences between smokers and non-smokers were found, except in the case of PN, significantly lower ratio in smokers, and in IPF, significantly higher ratio in smokers. Inversely to macrophages' results, total lymphocytes' frequency in smokers was decreased in comparison to non-smokers, being statistically significant in COPD and BA. Comparing the cell pattern of smokers and non-smokers with PD to the one of healthy smokers and non-smokers separately, it is easily understandable that the abnormal values observed in smokers are not only a PD outcome but also a cigarette smoke-related consequence. In conclusion, our results lead to the possibility that an apparently pathological value can be due to the subject's smoking habits and not due to a disease. Thereby, although preliminary, this study highlights the importance of considering the patient's smoking habits when interpreting the BAL cytometric analysis results in differential diagnosis of PD. 
P21

EVALUATION OF LYMPHOCYTIC PARAMETERS - VOLUME, CONDUCTIVITY AND SCATTER - IN A HEMATOLOGICAL ANALYZER IN PATIENTS WITH CHRONIC LYMPHOCYTIC LEUKEMIA

Micaela Batista, Jorge Reis, Bárbara Abreu, Hugo Macedo, Ana Paiva, Joana Diamantino, Frederico Valido

Clinical Pathology Department - Instituto Português de Oncologia de Coimbra Francisco Gentil, $E P E$

Introduction: Chronic Lymphocytic Leukemia (CLL) is the most common adult leukemia in western countries. CLL is characterized by a monoclonal B-cell count $\geq$ $5 \times 10^{9} / \mathrm{L}$ with characteristic peripheral blood morphology and immunophenotype. The VCS technology of Coulter hematology analyzers provide information about "positional parameters", such as volume (V), conductivity (C) and scatter (S) for each individual cell of white blood cell differential, that can be a comparable reflection of cell morphology.

Objective: Evaluate the lymphocyte positional parameters in patients with CLL.

Material and Methods: Blood cell counts were performed in 506 samples from patients with CLL and 42 samples from a healthy control group, using a Beckman Coulter LH-750 analyzer. Means and standard deviations of V, C and S of lymphocytes in both groups were evaluated and compared using Student T test. Pearson Correlation was used for compare the absolute number of lymphocytes in CLL with V, $\mathrm{C}$ and $\mathrm{S}$ parameters. Specificity and sensitivity were evaluated by analysis of the Receiver Operating Characteristic (ROC) curves. All statistical analysis was performed using IBM SPSS ${ }^{\circledR}$ Statistics v24.

Results: In CLL group, 56.32\% were males and the group age mean was 75,95 years. The mean values for V, C and S of lymphocytes observed were 76,60 $\pm 13,40$, $117,67 \pm 6,36$ and $66,85 \pm 10,25$, respectively. Whereas, in control group the values obtained for VCS were $86,19 \pm 2,83,113,30 \pm 2,51$ and $69,70 \pm 2,73$, respectively. Statistically significant difference were obtained between the two groups ( $\rho$ value $<0,05)$, regarding the three analyzed parameters (VCS). In CLL group, the absolute number of lymphocytes ranged between $10,10 \times 10^{9} / \mathrm{L}$ and $347,60 \times 10^{9} / \mathrm{L}$. In this group, we found a weak correlation between scatter and absolute lymphocyte counts $(r=0,324)$. ROC curves corresponding area under the curve (AUC) obtained for VCS were $0,782,0,256$ and 0,644 , respectively. Volume showed greater sensitivity $(83,6 \%)$ and specificity $(83,3 \%)$.

Conclusions: Our data suggest that lymphocyte positional parameters can be useful in differentiating pathological conditions such as CLL. Nonetheless, it would be interesting in an upcoming study to compare lymphocytic VCS parameters in other lymphoproliferative disorders and viral diseases. 
P22

HEPATITIS C VIRUS CORE ANTIGEN QUANTIFICATION AS AN ALTERNATIVE METHOD FOR INFECTION DIAGNOSIS

\author{
Daniela Gonçalves $^{1}$, Albertina Amaro ${ }^{1}$, Maria Conceição Faria², Débora Rodrigues ${ }^{2}$ \\ ${ }^{1}$ Universidade da Beira Interior, Covilhã, Portugal \\ ${ }^{2}$ Centro Hospitalar Universitário da Cova da Beira, Covilhã, Portugal
}

Background: Hepatitis $C$ is a public health problem as it remains the major cause of chronic liver disease. The diagnostic algorithm consists of two tests, HCV antibody test (HCV Ac) for screening and HCV RNA detection by real-time PCR for confirmation.

Objectives: Compare the performance of HCV core antigen (HCV Ag) quantification with HCV RNA detection in diagnosis of acute infection.

Materials/methods: A total of 39 stored serum samples from patients (ages between 27 and 78 years old) who were treated for HCV infection at a Portuguese university hospital were tested. HCV Ac was previously determined by electrochemiluminescent assay and HCV viral load was previously quantified by real-time PCR. The HCV Ag quantification test was performed by chemiluminescence.

Results: All 16 negative HCV RNA samples were negative for HCV Ag. In one HCV RNA positive sample with low viral load $(<15 \mathrm{IU} / \mathrm{ml}$ ) no HCV Ag was detected. A regression analysis of the $22 \mathrm{HCV}$ RNA positive samples with quantifiable HCV Ag produced the equation $y=0.662 x-0.671$. The correlation between $\log _{10} \mathrm{HCVAg}$ in $\mathrm{fmol} / \mathrm{L}$ and $\log _{10} \mathrm{HCV}$ RNA in IU/mL yielded a value of 0.972 , equally strong above the threshold $3000 \mathrm{UI} / \mathrm{mL}$ ( $r=0.962)$. The lowest HCV RNA quantification $(20.3 \mathrm{lU} / \mathrm{ml})$ yielded an HCV Ag quantification of $5.49 \mathrm{fmol} / \mathrm{L}$. HCV Ac previously tested positive in 30 of the 39 samples. For 9 patients there was no information on HCV Ac status.

Conclusions: All negative HCV RNA samples tested with HCV Ag showed negative results, demonstrating $100 \%$ concordance with RNA and high specificity of the test. The assay showed high sensibility in detecting HCV positive samples $(95,7 \%)$. In one sample with detectable RNA, but below the detection level of the assay it was not possible to detect HCV Ag, possibly due to interference caused by the presence of HCV Ac in the sample. The ability of the HCV Ag test to give a quantifiable result in samples with low viral load suggests that HCV Ag test could not only be used to diagnose infection but also to monitor the treatment. HCV Ag results can be issued within 2-3 days vs up to two weeks for real-time PCR results due to the necessity to batch samples for cost-effectiveness, enabling an earlier diagnosis and initiation of therapy. This study data show that HCV Ag test may be a simple and affordable tool for diagnosis and monitoring of HCV infection. 
P23

IS IT ADEQUATE FOR PATIENT CARE? micrOINR® POINT-OF-CARE SYSTEM EVALUATION IN PATIENTS ROUTINELY SEEN IN AN ANTICOAGULATION CLINIC

Graça Cunha Coutinho ${ }^{a}$ Isabel Alves Pereira ${ }^{a}$, Cristina Pita ${ }^{b}$, Cláudia Barão Ferreira ${ }^{a}$, Rosa Fula Bento ${ }^{a}$, Graça Cristina ${ }^{a}$

aUnidade Local de Saúde do Baixo Alentejo - Hospital José Joaquim Fernandes, ULSBA

${ }^{b}$ Centro Hospitalar Universitário do Algarve - Hospital de Portimão, CHUA

Introduction: Patients on vitamin $\mathrm{K}$ antagonists therapy (VKA) need frequent international normalized ratio (INR) monitoring. The use of point of care (POC) is extremely useful in patients who have difficult access to laboratory (LAB) and it is costeffective and less stressful. POC measure INR more rapidly. To access the reliability of $P O C$ devices it needs a rigorous evaluation.

The aim of this study was to evaluate the accuracy of the microlNR system for INR measurement in patients on VKA.

Methods: INR values in venous blood of 43 patients on VKA were obtained with Siemens BCS XP analyzer and compared with microINR values in capillary blood.

The data was analysed using MS Excel and IMB SPSS.

The measurement of accuracy was based on the CLSI EP09 protocol. Simple linear regression analysis was performed to compare the LAB INR with POC device values. The slope and intercept of the regression and the Bland-Altman plot were assessed. The quality of the data was assessed by evaluating the result ranges and the correlation coefficient.

Accuracy for each therapeutic interval was assessed according to acceptance criteria based on ISO 17593:2007. The dosage concordance (DC) - difference between methods $<0.5$ - and clinical agreement $(C A)$ were analysed.

Results: From the 43 patients on VKA 48.8\% were male and the average age was 73.8 \pm 9.3 years old. Correlation coefficient between microlNR and Siemens BCS XP was 0.951 ( $p<0.0001)$. Using the Bland-Altman analysis the mean difference was -0.14 , the standard deviation of the difference was 0.29 and the limits of agreement with $95 \%$ confidence level were -0.71 to 0.43 . The results within allowable difference (AD) for INR therapeutic interval ( $\geq 90 \%$ of results): for the group $<2.0$ ( $A D \pm 0.5$ ) was $90 \%$ and $100 \%$ for the groups between $2.0-4.5$ and $>4.5$ ( $A D \pm 30 \%$ ). The allowable bias $( \pm 0.3)$ in the group between $2.0-4.5$ was $-0,12$.

The DC was $93.0 \%$ and $95.3 \%$ for CA.

Conclusion: The microINR was found to be adequately accurate when compared with the validated $L A B$ method.

This POC has sufficiently high level of DC and CA to safely monitor the majority of patients on VKA, according with ranges reported from ISO 17593:2007 for similar devices. A limitation of the study is the small sample of patients mainly in supratherapeutic group, further study with a large sample is needed.

There are no conflicts of interest in all authors. 
P24

\section{CHARACTERIZATION OF CORYNEBACTERIUM SPECIES ISOLATES IN A TEN YEAR PERIOD}

Bruna Malheiro, Patrícia da Cunha Rodrigues, Alberta Faustino, Marina Majar, André Ferreira Pinto e Alexandra Estrada

Hospital de Braga

Introduction: Bacteria of the genus Corynebacterium have long been considered as contaminants, however studies have shown an increase in the number of isolates and their pathogenic potential.

Aims: The purpose of this study is to evaluate the frequency of Corynebacterium spp. isolates over a period of ten years, in a Hospital.

Material and Methods: We retrospectively studied Corynebacterium spp. isolates, non duplicated, in samples received for microbiological testing in the Laboratory of Clinical Pathology of a Hospital from January 2009 to December 2018. Identification of microorganisms was performed using the methods API Coryne ${ }^{\circledR}$, until 2016, MALDITOF $^{\circledR}$ from July 2017 and Vitek ${ }^{\circledR} 2$.

Results: During the study period, 443 strains of Corynebacterium were identified, of which 54,2\% were isolated in 2017 and 2018. Isolates were recovered in all age groups, with an average age of 61 years, $45.4 \%$ of which occurred in patients over 70 years old; $55,1 \%$ of the patients were male. The most commonly isolated species were C. striatum $(n=86), C$. amycolatum $(n=71), C$. jeikeium $(n=46), C$. pseudodiphtheriticum $(n=30)$ and C. propinquum $(n=27)$.

Over the ten-year study period, the specimens with a higher number of isolates were blood cultures ( $n=129)$, purulent exudates $(n=102)$ and respiratory samples $(n=72)$. Between 2009 and 2017, the specimens with more isolates yearly were blood cultures. In 2018, purulent exudates and respiratory samples were the most frequent, with $44,1 \%$ and $59,7 \%$ of the total isolates in these products occurring this year, respectively.

The species most frequently identified in blood cultures were $C$. jeikeium $(n=26)$; in purulent exudates $C$. amycolatum $(n=29)$ and $C$. striatum $(n=19)$; in respiratory samples $C$. striatum $(n=38)$ and $C$. pseudodiphtheriticum $(n=18)$. It should be noted that $84.6 \%$ of $C$. macginleyi isolates occurred in ocular exudates $(n=13)$. 
Conclusions: In this study, an increase in the number of isolates of Corynebacterium spp. was observed over ten years, which can be partly explained by the use of MALDITOF $^{\circledR}$, which allows an easier and more reliable species identification.

The species most frequently isolated in this study are in agreement with those described in the literature.

This increase should not be underestimated since many of the most frequently isolated species are known to be multiresistant.

References: Funke G, von Graevenitz A, Clarridge JE, 3rd, Bernard KA. Clinical microbiology of coryneform bacteria. Clinical microbiology reviews. 1997;10(1):125-59

Bernard K. The genus corynebacterium and other medically relevant coryneform-like bacteria. Journal of clinical microbiology. 2012;50(10):3152-8.

Yanai M, Ogasawasa M, Hayashi Y, Suzuki K, Takahashi H, Satomura A. Retrospective evaluation of the clinical characteristics associated with corynebacterium species bacteremia. The Brazilian Journal of Infectious Diseases. 2018;22(1):24-29 
P25

\section{ACTINOTIGNUM SCHAALII: AN UNDERESTIMATED URINARY TRACT INFECTION PATHOGEN}

Bruna Malheiro, Alberta Faustino, Marina Majar e Alexandra Estrada.

Hospital de Braga

Introduction: The genus Actinotignum contains three species, Actinotignum schaalii (formerly Actinobaculum schaalii), Actinotignum urinale and Actinotignum sanguinis. A. schaalii is part of the commensal flora of the genitourinary tract, however it is also involved in human infections, mostly urinary tract infections (UTI), particularly in elderly men and children.

Aims: In this study we present a case of an isolate of $A$. schaalii.

Materials and Methods: The microorganism was recovered from a urine sample. The sample was cultured in Columbia agar with $5 \%$ sheep blood $(\mathrm{COS})^{\circledR}$, Gelose Cystine Lactose Electrolyte Deficient (CLED) ${ }^{\circledR}$ and chocolate agar PolyviteX (PVX) ${ }^{\circledR}$, incubated at $35 \pm 2 \circ \mathrm{C}$ under $5 \% \mathrm{CO}_{2}$ atmosphere. Colony identification was performed using the MALDI-TOF ${ }^{\circledR}$ and Vitek ${ }^{\circledR} 2$ methodologies. The direct examination of the colonies was performed by Gram staining.

Results: Direct examination showed the presence of small coccoid rods, positive by Gram staining. In the microbiological culture, after 24 hours of incubation there was no growth in $\mathrm{CLED}^{\circledR}$ medium, but it was possible to observe growth in the $\operatorname{COS}^{\circledR}$ and $\mathrm{PVX}^{\circledR}$ media. Observation of the plates after 48 hours of incubation showed scarce growth in CLED ${ }^{\circledR}$ medium as well. The colonies observed were small, grey and nonhemolytic in $\operatorname{COS}^{\circledR}$. The microorganism was identified to the species level as Actinotignum schaalii using the Vitek $^{\circledR} 2$ and MALDI-TOF ${ }^{\circledR}$ (Bruker) methods.

Discussion / Conclusion: $A$. schaalii is often underestimated because it is a facultative anaerobe of slow and fastidious growth, requiring blood-enriched media and incubation under anaerobic or $5 \% \mathrm{CO}_{2}$ atmosphere for 48 hours. Because its colonies are small and grey, they may be wrongly considered contaminants or overgrown by other microorganisms. In this study, we were able to correctly identify this isolate using the MALDI-TOF ${ }^{\circledR}$ and Vitek ${ }^{\circledR} 2$ methods. Currently, the MALDI-TOF ${ }^{\circledR}$ Microflex Biotyper (Bruker) system is the fastest and most accurate method for routine identification of $A$. schaalii. Correct identification of this pathogen is important because strains are frequently resistant to co-trimoxazole and ciprofloxacin, which are widely used in the treatment of UTIs.

\section{References:}

Lotte, R., Lotte, L., \& Ruimy, R. (2016). Actinotignum schaalii (formerly Actinobaculum schaalii): a newly recognized pathogen-review of the literature. Clinical Microbiology and Infection, 22(1), 28-36. https://doi.org/10.1016/j.cmi.2015.10.038

Lotte, L., Lotte, R., Durand, M., Degand, N., Ambrosetti, D., Michiels, J.-F., Ruimy, R. (2016). Infections related to Actinotignum schaalii (formerly Actinobaculum schaalii ): a 3-year prospective observational study on 50 cases. Clinical Microbiology and Infection, 22(4), 388-390. https://doi.org/10.1016/j.cmi.2015.10.030 
P26

\section{DETERMINATION OF THE ENZYME PHENOTYPE THIOPURINE S- METHYLTRANSFERASE BY HIGH PERFORMANCE LIQUID CHROMATOGRAPHY - IMPLEMENTATION IN THE HOSPITAL ROUTINE}

Catarina Sousa Neves ${ }^{1}$, Daniela Fonseca e Silva ${ }^{1}$, Luísa Carvalho ${ }^{1}$, Isabel Silva ${ }^{1}$, Henrique Reguengo ${ }^{1}$, José Carlos Oliveira ${ }^{1}$

${ }^{1}$ Serviço de Química Clínica, Centro Hospitalar e Universitário do Porto

Introduction: Thiopurine S-methyltransferase (TPMT) is one of the enzymes responsible for the metabolism of thiopurine drugs by converting those into inactive metabolites. Examples of these drugs are Mercaptopurine, Azathioprine and Thioguanine. Their metabolism is strongly affected by genetic polymorphisms which can cause a wide variation between the efficacy and toxicity of thiopurine drugs being the main side effect severe myelosuppression ${ }^{1}$. The purpose of this study was to determine the activity of TPMT of patients who were to initiate thiopurine drugs and to identify the origin of these requests and the attributed diagnosis. As to our knowledge we are the first tertiary hospital in Portugal to initiate this enzyme determination.

Material and methods: Implementation of an assay using a high performance liquid chromatography method (Alliance Waters $2695^{\circledR}$ ) with fluorescence detector (FP-920 Jasco $^{\circledR}$ ). The technique developed was based on studies from the Birmingham group ${ }^{2}$. The type of sample used is whole blood collected in a blood cell tube containing EDTA tripotassium. The sample undergoes an enzimatic treatment before being injected into the chromatography equipment.

Results: The study included 55 patients (26 females and 29 males) from 09/02/2018 to $04 / 12 / 2018$ with an average age of 36,43 years. Requests were made from Dermatology, Gastroenterology, Hematology and Internal Medicine. The majority of diagnosis that motivated the requests were auto immune diseases: Crohn Disease, Ulcerative Colitis, Inflammatory Bowel Disease, Autoimmune Hepatitis, Vulgar Pemphigus and Chronic Immune Thrombocytopenia. Regarding the phenotype results: $29 \%(n=16)$ exhibited low activity of TPMT $(10-69 \mathrm{mU} / \mathrm{L})$ and $71 \%(\mathrm{n}=39)$ exhibited normal TPMT activity $(70-150 \mathrm{mU} / \mathrm{L})$ according to the reference values of the Birmingham group.

Conclusion: Most requests were made regarding patients with diverse auto immune diseases prior to the utilization of thiopurine drugs. The majority of the results indicated a wild type TPMT phenotype and there were no extreme results for TPMT activity. The determination of the phenotype of TPMT is an important analysis that allows the optimization of pharmacotherapy based on the individual profile.

\footnotetext{
${ }^{1}$ Loretta Ford, Valerie Graham and Jonathan Berg. Whole-blood thiopurine S-methyltransferase activity with genotype concordance: a new, simplified phenotyping assay Ann Clin Biochem 2006; 43: 354-360 ${ }^{2}$ Sandwell and West Birmingham Hospitals NHS Trust. Thiopurine S-Methyltransferase Activity in Whole Blood (mU/L)
} 
P27

DENGUE FEVER - THE MIMICKER

Marco P. Barros Pinto, Richard Vanegas, Guilhermina Gaião Marques, Cristina Duarte

Hospital de Santa Maria - Centro Hospitalar Universitário Lisboa Norte, EPE

Introduction: Dengue is an infectious disease that is caused by dengue virus. Transmission takes place through bites by Aedes mosquito. Clinical manifestations vary from mild undifferentiated febrile illness to severe dengue, including dengue hemorrhagic fever and dengue shock syndrome, the latter is the most live threatening. One of the characteristic features of dengue is the occurrence of leucopenia and thrombocytopenia, probably resulting from virus-induced bone marrow suppression. Despite the general bone marrow suppression, blood plasmacytosis has been reported in a few patients with dengue infection. Plasmocytosis in peripheral blood is a rare finding more often associated with plasma cell leukemia or advanced stage multiple myeloma (in which case the plasma cells are part of the malignant clone and thus are monoclonal) but rarely with other malignancies, infectious diseases (acute respiratory infections, parvovirus $B 19$, rubella and hepatitis $A$ ), sepsis, autoimmune disorders or drug reactions.

Case Report: We report the case of a 40 year-old man who travelled to Brazil. He turner to the hospital with fever, headache, anorexia and sweating for 5 days. No hemorrhagic dyscrasias signs were identified. An initial evaluation revealed elevated

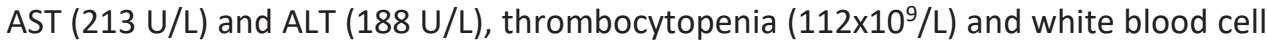
count of $7.62 \times 10^{9} / \mathrm{L}$. In the peripheral blood smear were identified $10 \%$ plasmacytoid lymphocytes/plasma cells, $43 \%$ neutrophils, $1 \%$ eosinophils, $43 \%$ lymphocytes and $3 \%$ monocytes. Serologic results were reactive for dengue (immunoglobulin M [IgM] positive).

After treatment the patient recovered from his disease and in the reassessment exams a peripheral blood smear was performed and no plasmacytoid lymphocytes/plasma cells were found.

Discussion: Epidemic dengue fever has recently reemerged worldwide, so an associated plasmacytosis may be seen more frequently. In dengue fever, peripheral blood smears may show the presence of plasmacytosis. If due to dengue fever, it should resolve with resolution of the disease. Recognition that it is a self-limited phenomenon could avoid unnecessary diagnostic investigation. 
P28

IS THIS BLOOD COUNT REALLY URGENT? WHAT THE NUMBERS TELL US.

Marco P. Barros Pinto, Cristina Vaz Carneiro, Guilhermina Gaião Marques

Hospital de Santa Maria - Centro Hospitalar Universitário Lisboa Norte, EPE

Introduction: Quality in laboratory (lab) medicine must assure valuable medical decision making.

The pre-analytical errors account for up to $70 \%$ of all errors made in lab, most of which arise from problems in patient preparation, sample collection, transportation, and preparation for analysis and storage.

A considerable proportion of the budget in hospitals is increasingly spend on diagnostic tools such as lab tests. Doctors are awareness about this issue, but they tend to pay much attention to reduction in the amount of so called expensive tests and little attention to cheap tests which are performed frequently.

Emergency care services must be fast, with an expected target time of 4 hours in the most benign situations (blue bracelet), according with the Manchester triage.

The blood count plays an important role in the diagnosis of a significant group of diseases, describing by Direção Geral de Saúde in the Norma 63/2011 its prescription roles. But how often is it requested at the emergency department without being essential for making an urgent diagnostic and / or therapeutic decision?

Statistical Analysis: Starting from 200 rejected blood samples in the lab due to the presence of a clot, the time elapsed between the communication of this finding to the emergency department, the request and resubmission of a new sample was evaluated prospectively, seeking to ascertain the real need of the blood count for the medical decision. It was considered that the maximum time for resubmission of a new sample to the lab, when it is essential for clinical and/or therapeutic decision-making, should not exceed 4 hours.

The time of resubmission of a new sample was divided into 5 groups: $\leq 1$ hour; ] 1-4] hours; ] 4-12] hours; ] 12-24] hours and > 24 hours.

The analysis of the data shows us that $25.5 \%$ of the resends occurred in $\leq 1$ hour; $16.5 \%$ between ] 1-4] hours; $11 \%$ between ] 4-12] hours; $16 \%$ between ] 12-24] hours and $31 \%$ at $>24$ hours.

Discussion: Only $42 \%$ of the requested blood counts were essential to take an urgent diagnostic and/or therapeutic decision. Thirty-one percent of the blood counts were not needed for decision making, having been resent to the lab in more than 24 hours or never been resent to lab.

These results highlight the importance of increasing the awareness among the clinicians about the importance of reducing unnecessary tests. 
P29

ANALYSIS OF VITAMIN D LEVELS IN 2018: A RETROSPECTIVE STUDY

Cristiana Reis, Rui Teixeira, Emília Krasteva, Mariana Feijó, Sara Correia, Maria Conceição Faria

Universidade da Beira Interior

25-hydroxycholecalciferol (Vitamin D) is an liposoluble hormone that plays a key role in the regulation of several organs and tissues. The importance of vitamin $D$ together with its increasingly reported lower levels becomes a global concern. In laboratorial routine, exact measurements of Vitamin D and methodological standardization are essential to avoid quantification errors and to assure results reproducibility. Thereby, the evaluation and critical analysis of these results are extremely important. The present work aims to analyze all of Vitamin D measurements performed in our institution, over the last year.

During 2018, 3100 patients' serum samples were analyzed through a chemiluminescent microparticle immunoassay realized in Cobas $^{\circledR} 6000$ equipment (Roche). Study population ages are comprised between 1 and 101 years, with the average age of 62 years, being that $62,3 \%$ were females. Vitamin D levels, gender, age, month of analysis and specialty which requires the analysis were defined as study variables. Statistically significant differences between groups were analyzed by Kruskal-Wallis, Mann Whitney and Pearson's correlation tests, utilizing IBM SPSS Statistics (version 25) software.

This retrospective analysis demonstrated that the majority of the studied population presented deficient ( $\leq 20 \mathrm{ng} / \mathrm{mL}, 56,8 \%$ ) or insuficient $(21-29 \mathrm{ng} / \mathrm{mL}, 30,7 \%)$ vitamin $D$ levels, being that only $12,5 \%$ presented sufficient levels. A negative correlation ( $p<$ 0,001 ) between age and vitamin $D$ was demonstrated, since vitamin $D$ levels decrease with age. Even more, this result was shown to be gender-independent. Despite of Vitamin $D$ measurement, requirements were essentially from consultation $(83,5 \%)$, statistically significant $(p<0,001)$ lowest levels of vitamin $D$ were observed in internment patients. Medical $(38,9 \%)$, rheumatology $(12,3 \%)$, and diabetology $(9,5 \%)$ were the specialties that more frequently required vitamin $D$ measurements. The month when the measurement was performed significantly influences the vitamin $D$ levels $(p<0,001)$, being the highest levels observed in September and lowest in December.

In conclusion, vitamin D low levels were observed in almost every one of the study subjects, being that even on the months with higher sun exposition this deficit was observed. 
P30

DETERMINATION OF URINARY CORTISOL IN CUSHING SYNDROME, OBESITY AND CONTROL POPULATION: COMPARISON WITH THE EXPECTED VALUES OF THE METHOD IN USE

Conceição Godinho $^{2}$; Ana Claudia Martins ${ }^{1}$; Isaura Rodrigues ${ }^{2}$; Susana Prazeres ${ }^{3}$, Deolinda Madureira ${ }^{4}$

${ }^{1}$ Serviço Endocrinologia do Centro Hospitalar Universitário Lisboa Central

2 Serviço de Patologia Clinica do Centro Hospitalar Universitário Lisboa Central

${ }^{3}$ Serviço de Patologia Clinica Instituto Português de Oncologia Lisboa

${ }^{4}$ Responsável pelo GELEND (Grupo de Estudos de Laboratório Sociedade Portuguesa de

Endocrinologia Diabetes e Metabolismo SPEDM)

Introduction: Free cortisol excretion in 24 hour urine (UFC), is one of the recommended tests in the initial investigation of Cushing's syndrome (CS). It is essential to interpret the results in the context of the appropriate range. Clinicallaboratory validation of the expected values proposed by the manufacturer reinforces its usefulness.

Objectives: To determine UFC in 3 groups: patients with diagnosis of CS (Group 1), patients with obesity (Group 2) and in a control population composed of individuals with low probability of hypo / hypercortisolism (Group 3). It is intended to compare UFC between the different groups according to expected values proposed by the manufacturer.

Material and Methods: 2722 evaluations of UFC were determined in Architect-Abbott (CMIA), between 28-2-2015 and 30-9-2018; expected values 4.3-176 $\mu \mathrm{g} / 24 \mathrm{~h}$ ( $\mathrm{n}=$ 128), slope $1.0 \pm 0.2$ and $r \geq 0.85$ when compared to LC-MS / MS, according to manufacturer's information. 181 determinations were excluded for urinary volume $<800 \mathrm{ml}$ and 164 for lack of clinical information. Diagnosis of CS was based on clinical process information and other laboratory tests (serum and salivary cortisol, ACTH, dexamethasone suppression tests). Control group was defined after exclusion of hospitalized patients and clinical situations that could condition hypo or hypercortisolism. Statistical analysis: Microsoft Excel and SPSS. Measurements below the detection limit of the assay $(<0.4 \mu \mathrm{g} / \mathrm{dL})$ or extreme values were not considered. Results: 56 UFC in Group 1 (32 patients), 820 in Group 2 (799 patients) and 365 in Group 3 (354 patients). Group 1 - mean $97 \pm 94.8 \mu \mathrm{g} / 24 \mathrm{~h}$, median $68.8 \mu \mathrm{g} / 24 \mathrm{~h}$, min-

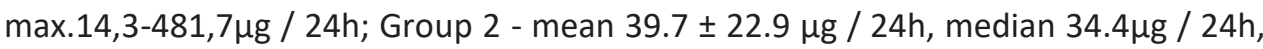

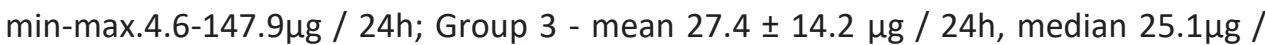

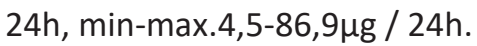

Conclusions: Statistically significant differences were found between the distribution and median UFC in the 3 groups studied $(p<0.05)$. UFC in the control group was lower than the proposed values. These results show the importance of the validation of the expected values proposed by the manufacturer, which should be adapted to the population. 
P31

\title{
DIAGNOSTIC ACUITY OF FREE URINARY CORTISOL IN CUSHING SYNDROME: A QUESTION OF EXPECTED VALUES?
}

\author{
Conceição Godinho ${ }^{2}$; Ana Claudia Martins; Isaura Rodrigues²; Susana Prazeres ${ }^{3}$, \\ Deolinda Madureira ${ }^{4}$ \\ ${ }_{1}^{1}$ Serviço Endocrinologia do Centro Hospitalar Universitário Lisboa Central \\ ${ }^{2}$ Serviço de Patologia Clinica do Centro Hospitalar Universitário Lisboa Central \\ ${ }^{3}$ Serviço de Patologia Clinica Instituto Português de Oncologia Lisboa \\ ${ }^{4}$ Responsável pelo GELEND (Grupo de Estudos de Laboratório Sociedade Portuguesa de \\ Endocrinologia Diabetes e Metabolismo SPEDM)
}

Introduction: In the suspicion of Cushing's syndrome (CS), the evaluation of free urinary cortisol in 24 hours (UFC) is one of the recommended tests. Clinical-laboratory validation of the expected values proposed by the manufacturer reinforces its usefulness.

Objectives: To compare UFC in CS patients and in a control group composed of individuals with low probability of hypo / hypercortisolism. To determine values of cut-off (clinical sensitivity and specificity) according to the data of the study population

Material and Methods: 2722 evaluations of UFC were determined in Architect-Abbott (CMIA), between 28-2-2015 and 30-9-2018; expected values 4.3-176 $\mu \mathrm{g} / 24 \mathrm{~h}$ ( $\mathrm{n}=$ 128). Based on clinical information, 2 groups were selected. Group 1 (CS) and Group 2 (Control group) was defined after exclusion of hospitalized patients and clinical situations that could condition hypo / hypercortisolism. Statistical analysis: Microsoft Excel and SPSS. Measurements below the detection limit of the assay or extreme values were not considered. ROC curve analysis: sensitivity and specificity at different cut-offs were obtained for which the UFC concentration is considered highly suggestive of hypercortisolism.

Results: UFC concentrations: CS Group 1 - mean $97 \pm 94.8 \mu \mathrm{g} / 24 \mathrm{~h}$, median $68.8 \mu \mathrm{g} /$ 24h, min-max. 14,3-481,7 ( $n=56)$; Control Group 2 - mean $27.4 \pm 14.2 \mu \mathrm{g} / 24 \mathrm{~h}$, median $25.1 \mu \mathrm{g} / 24 \mathrm{~h}$, min-max. $4,5-86,9(\mathrm{n}=365) .95 \%$ of the population studied (control group) corresponds to $8-63.3 \mu \mathrm{g} / 24 \mathrm{~h}$. ROC curve for UFC of the 2 groups: AUC $=0.85$. ROC curve analysis: Sensitivity and specificity at different cutoffs: $176 \mu \mathrm{g} / 24 \mathrm{~h}-18.8 \%$ and $100 \% ; 63.3 \mu \mathrm{g} / 24 \mathrm{~h}-59.4 \%$ and $97.5 \%$ respectively.

Conclusions: The cut-off based on the expected values proposed by the manufacturer, despite having a specificity of $100 \%$, has a low sensitivity $(18.8 \%)$, leading to a high false negative rate, which is unsuitable for a screening test. The cut-off calculated for the control group showed a better sensitivity (59.4\%), maintaining high specificity. In order to improve the diagnostic acuity in CS screening, the authors propose the establishment of expected values healthy population-based, in order to calculate the cut-off with better sensitivity and specificity in Cushing's syndrome screening. 
P32

\section{A RELAPSE OF CUSHING DISEASE: A CASE REPORT}

Authors: Margarida Pereira ${ }^{1}$, Nuno Gonçalves ${ }^{2}$, Anabela Leão ${ }^{2}$, Ana Paula Santos ${ }^{3}$, Isabel Torres ${ }^{3}$, Luis Araújo ${ }^{2}$

${ }^{1}$ Clinical Pathology Department, Unidade Local de Saúde de Matosinhos EPE

${ }^{2}$ Clinical Chemistry Department, Instituto Português de Oncologia do Porto Francisco Gentil EPE

${ }^{3}$ Endocrinology Department, Instituto Português de Oncologia do Porto Francisco Gentil EPE

Introduction: Cushing's disease (CD) is associated with marked morbidity and mortality thus definitive treatment is mandatory which requires localization of ACTH source. Bilateral inferior petrosal sinus sampling (BIPSS) is considered as gold standard in differentiating pituitary dependent CD from ectopic CD. BIPSS is an interventional radiology procedure in which ACTH levels obtained from venous drainage very near the pituitary gland are compared to peripheral blood levels before and after corticotropin-releasing hormone $(\mathrm{CRH})$ stimulation.

Case report: A 40-year-old woman presented with CD in 2010 underwent a transsphenoidal adenomectomy. Pathohistological evaluation suggested a macroadenoma. 7 years later laboratory findings revealed increased level of cortisol [serum cortisol 08:00h 49,02 ug/dL (normal range 6,2-19,4 ug/dL), 17:00h 51,57 ug/dL (normal range 2,3-11,9 ug/dL] with loss of circadian rhythm. Levels of ACTH were normal $(32,79 \mathrm{pg} / \mathrm{dL}$, normal range $7,2-63,3 \mathrm{pg} / \mathrm{mL})$. Urinary free cortisol was increased $(1139,6 \mathrm{ug} / 24 \mathrm{~h}$, normal range 36-137 ug/24 h), as also midnight salivary cortisol level (1,05 ug/dL, normal range $<0,32 \mathrm{ug} / \mathrm{dL}$ ) A overnight dexamethasone suppression test showed insensitivity to dexamethasone suppression (serum cortisol 08:00h 11,56 ug/dL, normal range < $5.0 \mathrm{ug} / \mathrm{dL}$ ). Imaging studies with MRI brain did not reveal any pituitary adenoma. In view of non-localization of source of ACTH BIPPS were performed.

ACTH values were used to calculate the ratio of ACTH between the right or left inferior petrosal sinus and the concentration in the peripheral blood (IPS:P ratio) and the maximal ratio (right or left) was calculated. Basal IPS/PV ratio of ACTH $>2$, and post stimulation IPS/PV > 3 were suggestive of pituitary dependent $C D$. Interpetrosal ratio of $\mathrm{ACTH}<1,5$ showed no lateralization of pituitary adenoma.

Discussion: The diagnosis for CD is made by laboratory testing, which demonstrates the consistent overproduction of cortisol.

This case report highlights the importance of long-term patient monitoring in CD and the importance of BIPSS to establish the diagnosis of ACTH-dependent Cushing's disease if no obvious tumor is seen in MRI. BIPSS have an overall sensitivity and specificity of $96 \%$ and $100 \%$ respectively. 
P33

\title{
LABORATORY EVALUATION OF PROTEINURIA
}

\author{
Isabel Cachapuz \\ Clinical Pathology Department, Unidade Local de Saúde de Matosinhos
}

Introduction: Mean daily urinary excretion of proteins in adults is $80 \mathrm{mg} /$ day and normal excretion is $<150 \mathrm{mg} /$ day. Proteinuria (PT) varies in quantity and may be transient or persistent.

Objectives: Review main pathophysiological mechanisms of PT and methods currently used in its detection and quantification. Verify clinical importance and present a protocol of diagnostic approach.

Material and Methods: Including 68 articles published in last 5 years of original articles, review and meta-analyzes.

Results: By pathophysiology, PT is classified: glomerular (PG), tubular (PT) and overload (PS). PG arises by increasing the filtration of macromolecules in the glomerular filtration barrier. PT is composed of low molecular weight proteins. PS is due to presence of high plasma protein concentrations which is filtered at glomerular filtration barrier but exceeds the capacity for proximal tubular reabsorption - usually associated with Bence-Jones Proteinuria. Methods for PT detection and quantification: 1) Test and Exam Strips Urine Summary; 2) Test strips for the detection of microalbuminuria; 3) Proteinuria of the $24 \mathrm{~h}$; 4) Ratio Protein / creatinine in random sample 5) Albumin / Protein ratio in random sample 6) Urinary Protein Electrophoresis; 7) Immunoelectrophoresis of Urinary Proteins. Dagnostic algorithm for PT consists: When detected in a routine analysis (Test Strips / Urine Summary) should be repeated 2 to 3 times before proceeding with study. Complete clinical history. If PT remains on repeat or 3+ to 4+ analyzes at the initial routine examination, etiological investigation should continue with 24-hour PT or Ratio albumin / creatine or Protein / creatinine in a random urine sample. If PS is suspected, for qualitative evaluation of PT, perform urinary protein electrophoresis and if monoclonal peak identify chain type by urinary immunoelectrophoresis.

Conclusion: Clinical significance of PT is variable. The use of diagnostic algorithm such as the one described helps clinical distinguish more frequent and benign causes of the rarer and pathological causes. When cause remains unknown, patients should be referred to Nephrologist. Incidence of CKD has been rising, so diagnosis and monitoring of risk factors are important. 
P34

HYPOPHOSPHATEMIA: INVESTIGATION A CASE REPORT

\author{
Margarida Pereira ${ }^{1}$, Ivo Cunha ${ }^{2}$, Sofia Bastos ${ }^{1}$, Sandra Silva ${ }^{3}$, Isabel Cachapuz ${ }^{1}$ \\ ${ }^{1}$ Clinical Pathology Department, Unidade Local de Saúde de Matosinhos EPE \\ ${ }^{2}$ Internal Medicine Department, Unidade Local de Saúde de Matosinhos EPE \\ ${ }^{3}$ Nephology Department, Unidade Local de Saúde de Matosinhos EPE
}

Introduction: The concentration of serum phosphate $(\mathrm{Po})$ depends on intestinal absorption, renal handling and skeletal storage; consequently, is it regulated by parathyroid hormone, vitamin D, and fibroblast growth factor (FGF) 23.

Case report: A 39 years woman was diagnosed with iron deficiency anaemia. The diagnostic workup, including for celiac disease were negative and she underwent $1 \mathrm{~g}$ of intravenous(iv) ferric carboxymaltose. Her past medical history was relevant for a total thyroidectomy in 2015 and her lab results showed mild hypocalcaemia (hypoCa) and no other abnormalities besides anaemia.

3 weeks later she complained of with fatigue, diffuse muscle pain and weakness. Laboratory findings showed hypophosphatemia (hypoPo) and hypoCa and she started oral suplementation. Clinical picture got worse, with numbness and paresthesias and severe hypoPo $(1 \mathrm{mg} / \mathrm{dl})$ and low ionized calcium $(0,8 \mathrm{mg} / \mathrm{dl})$ were identified. PTH, D vitamin, magnesium, CK and liver enzymes were normal. She was hospitalized for iv supplementation and access of the etiology of ionic disorders. 24 hours urinary Po was 1,31g and fractional excretion of filtered phosphate (FEPO4) 20\%. 6 days later she was discharge with relieve of symptomatology and normalization of ionic levels.

Discussion: Prolonged hypoCa with a low normal PTH goes in favor of primary hypoparathyroidism probable due to total thyroidectomy. PTH is not released in hypoCa that normally trigger PTH release.

About hypoPo, spurious hypoPo with the Po assay was eliminated. A 24-hour urine Po excretion $>100 \mathrm{mg}$ and FEPO4 $>5 \%$ indicates renal phosphate wasting, which may be due to hyperparathyroidism, increased FDG 23 levels, or a primary renal tubular process such as Fanconi syndrome. The absence of albuminuria and glucosuria ruled out a Fanconi syndrome. Chronic FGF23 excess as in tumour-induced osteomalacia is usually associated to increased bone-specific alkaline phosphatase. It's described in literature that administration of ferric carboxymaltose inhibits FGF23 degradation and thereby increases FGF23, which then can cause hypoPo that could be an explanation to this case. FGF23 assays are not routinely available and expensive.

Considering the expanding use of iv iron, we hope our case contributes to the awareness of clinicians for this potential complication. 
P35

ATYPICAL CML - THE IMPORTANCE OF MORPHOLOGY

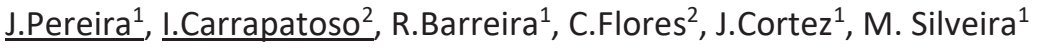 \\ 1 Instituto Português de Oncologia de Lisboa Francisco Gentil - Laboratório de Hematologia, \\ Serviço de Patologia Clínica \\ ${ }^{2}$ Centro Hospitalar Universitário Lisboa Central - Serviço de Patologia Clínica
}

Introduction: Atypical chronic myeloid leukaemia (aCML), BCR-ABL1-negative is a rare haematological neoplasm recognised by the World Health Organisation (WHO 2016) classification belonging to the group of myelodysplastic/myeloproliferative (MDS/MPN) syndromes and largely defined morphologically. It's characterised by leukocytosis and prominent granulocytic dysplasia. WHO diagnostic criteria that can help distinguish aCML from other chronic leukaemias include: persistent leukocytosis ( $\geq 13 \times 109 / 1), \geq 10 \%$ immature myeloid cells (promyelocytes, myelocytes, and metamyelocytes) in the peripheral blood and/or dysplasia, absent or minimally present basophilia ( $<2 \%$ of leukocytes) and absent or minimally present monocytosis ( $<10 \%$ of leukocytes). aCML is associated with SETBP1 and/or ETNK1 mutations in up to a third of cases.

Clinical Case: A 71 year old angolan man presented at the emergency department with haematuria beginning two days ago associated with asthenia for some weeks. Analytically he presented with anaemia, thrombocytopenia and immature myeloid cells on the peripheral blood smear (PBS). The patient was sent to our institution with the probable diagnosis of a chronic myeloproliferative neoplasm. At admission he presented the following blood count: haemoglobin 7,2g/L; leucocytes 57,60x109/L; platelets $49 \times 109 / \mathrm{L}$. Given the clinical suspicion and the laboratory results we performed a PBS, a bone marrow aspiration and trephine biopsy and proceeded to the cytomorphologic, cytogenetic and immunophenotypic analysis.

PBS: $18 \%$ myeloblasts, $2 \%$ promyelocytes, $13 \%$ myelocytes, $23 \%$ metamyelocytes, $25 \%$ neutrophiles; $11 \%$ lymphocytes $3 \%$ monocytes; $2 \%$ basophiles. With prominent granulocytic dysplasia.

Cytomorphology of bone marrow: hypercellular, with granulocytic hyperplasia, an increased number of myeloblasts (<20\%) and prominent dysgranulopoiesis: hypogranulation; maturative asynchronisms; mild dyseritropoiesis; absent megakaryocytic dysplasia.

Cytogenetics: $\mathrm{t}(9 ; 22)(\mathrm{q} 34 ; \mathrm{q} 11)$ negative; atypical hybridation pattern: $81 \%$ of cells signals of atypical hybridation to the ABL1 gene; Karyotype $48, X Y,+8,+9$. BCR-ABL negative. JAK2 negative. NPM1 negative. CALR negative.

Flow cytometry: Myeloid lineage with $18 \%$ myeloblasts and $61 \%$ mature cells, hypogranulated, dysplasic, showing phenotypic distortions evaluated by CD11b/CD13.

Discussion/Conclusion: Given the above mentioned results the diagnosis of atypical chronic myeloid leukemia, BCR-ABL1-negative was admitted. This case shows the importance of a correct laboratory cytomorphologic and cytogenetic diagnosis in order to distinguish aCML from other myeloid neoplasms with similar findings. 
P36

MISFORTUNES NEVER COME SINGLY: HCV, HEPATOCELLULAR CARCINOMA AND MANTLE CELL LYMPHOMA

Marco P. Barros Pinto, Cristina Duarte

Hospital de Santa Maria - Centro Hospitalar Universitário Lisboa Norte, EPE

Introduction: Hepatitis C virus ( $\underline{\mathrm{HCV}}$ ) infection frequently leads to chronic hepatitis and is a major cause for hepatocellular carcinoma (HCC). Hematological manifestations such as type II mixed cryoglobulinemia or B-cell non-Hodgkin lymphoma ( $\underline{\mathbf{B}-\mathrm{NHL}}$ ) are less common.

Cumulative epidemiologic evidence supports the thesis that patients with chronic HCV infection are at increased risk to develop lymphoma. The most convincing evidence for a causal relationship between HCV infection and lymphoma development is the observation of B-NHL regression after HCV eradication by antiviral therapy.

$\mathrm{B}-\mathrm{NHL}$ is strongly associated with HCV that was proved in the last 2 decades.

The molecular mechanisms of HCV-associated lymphomagenesis are still poorly understood. Three general theories have emerged to understand the HCV-induced lymphomagenesis: 1) continuous external stimulation of lymphocyte receptors by viral antigens and consecutive proliferation; 2) HCV replication in B cells with oncogenic effect mediated by intracellular viral proteins; 3 ) permanent B-cell damage, e.g., mutation of tumor suppressor genes, caused by a transiently intracellular virus.

B-NHL subtypes most frequently associated with HCV are marginal zone lymphoma and diffuse large B-cell lymphoma.

Mantle cell lymphoma ( $\underline{\mathbf{M C L}}$ ) is a rare subgroup of B-NHL that occurs in approximately $6 \%$ of all NHL patients.

Case Report: A 72-years-old man with a personal history of HCV and HCC (hepatectomy in 2006), come in to the hospital at the end of 2018 due to the presence of a left retroauricular mass with 4 weeks evolution and a $5 \mathrm{~kg}$ weight loss in the last 3 weeks. In the course of the clinical investigation, a bone marrow aspiration was perform, revealing the presence of infiltration by blast cells ( $99 \%$ of cells). Immunophenotyping by flow cytometry identified a monoclonal lymphoid B population (CD20+/CD5+/CD10-/CD43-/CD200-/CD38-/CD79b+/Kappa +) compatible with mature B-cells neoplasia/B-NHL CD5+. FISH was positive for $t(11,14)(q 13 q 32)$ IgH/CCND1 in $90 \%$ of analyzed cells. These findings are compatible with MCL. Patient started treatment.

Discussion: Hepatitis C virus infection is a major cause for HCC and less often for BNHL. This case report illustrates this relationship, highlighting the importance of surveillance of patients with HCV infection not only for HCC but also for B-NHL. 
P37

HAEMATOLOGY AND BIOCHEMISTRY PARAMETERS IN DE NOVO ATRIAL FIBRILLATION FOLLOWING AORTIC VALVE REPLACEMENT SURGERY - A PAIRED ANALYSIS

Fragão-Marques M, MD MSc ${ }^{1,2}$; Oliveira PJ, BSc ${ }^{1}$; Manco C, MD MSc; Oliveira R, MD MSc; Lourenco AP, MD PhD ${ }^{1}$; Falcão-Pires I, $\mathrm{PhD}^{1}$; Leite-Moreira AF, MD PhD ${ }^{1}$

${ }^{1}$ Department of Surgery and Physiology, Faculty of Medicine of the University of Porto, Portugal ${ }^{2}$ Department of Clinical Pathology, São João University Hospital, Porto, Portugal

Objectives: To assess cardiovascular biomarkers of postoperative atrial fibrillation (POAF) based on haematological and biochemical parameters and evaluate the progression of such biomarkers from a preoperative status to a postoperative setting.

Materials and methods: Patients submitted to aortic valve replacement in 2014 and 2015 were selected and their clinical and analytical data retrospectively collected. Selected variables had a normal or binomial distribution, therefore univariate analysis by group (postoperative atrial fibrillation - POAF vs. postoperative sinus rhythm POSR) was performed using t-test and chi-squared for continuous or dichotomous variables, respectively. Furthermore, a paired t-test analysis was used to compare postoperative analytical parameters in relation to their baseline values. Logistic regression was conducted in order to test for plasmatic biomarkers as independent predictors of the arrhythmia.

Results: 379 patients were included, of which $41.2 \%$ had de novo postoperative atrial fibrillation, with a mean occurrence at $2.5 \pm 1.5$ days. Age was significantly associated with postoperative atrial fibrillation $(p=<0.001)$. Inflammatory biomarkers such as $C$ Reactive Protein and total leukocyte count were tested, although only the former associated with the arrhythmia $(p=0.015)$. A logistic regression model was tested and suggested age and postoperative C-Reactive Protein (CRP) peak as independent predictors of POAF ( $p=0.002$ and $p=0.038$, respectively). The calculated Area Under the Curve $(A \cup C)$ for the predicted probabilities was 0.7 . In the paired analysis, every haematological parameter differed in the postoperative setting in relation to baseline, regardless of the patient subgroup. Creatinine clearance was the only parameter which was altered only in POAF patients $(p<0.001)$. Furthermore, total leukocyte count and creatinine clearance had a more marked postoperative alteration in POAF when compared to POSR patients.

Discussion/Conclusions: POAF is an extremely frequent and relevant medical condition with both clinical and financial adverse outcomes. These results suggest POAF patients are more prone to postoperative renal dysfunction and have a marked inflammatory response. CRP emerges as a potential analytical biomarker for de novo postoperative atrial fibrillation in aortic stenosis patients. 
P38

\title{
COMPARATIVE STUDY OF TWO TESTS FOR DETERMINATION OF FREE LIGHT
} CHAINS.

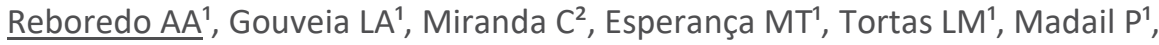 \\ Ramalheira $\mathrm{E}^{1}$ \\ ${ }^{1}$ Serviço de Patologia Clínica do Centro Hospitalar do Baixo Vouga. \\ ${ }^{2}$ Instituto Superior de Contabilidade e Administração da Universidade de Aveiro.
}

Introduction: Free light chain (FLC) analysis by serum immunoassay used since the beginning of the century became an important criteria in the study of monoclonal gammopathies due to the latest updates made by IMWG (International Myeloma Working Group).

Goals: Recent studies have used Freelite ${ }^{\circledR}$ reagents (The Binding Site). There are now other methods such as N Latex FLC $^{\circledR}$ (Siemens).

The aim of this study was to evaluate the similarity of both tests, verifying the presence or absence of significant differences.

Material and Methods: 104 serum samples with monoclonal bands demonstrated by electrophoresis and immunofixation from the laboratory routine between February and September 2018 were analyzed.

The BN ProSpec nephelometer (Siemens) was used in parallel analysis of the reagents under study: Freelite (polyclonal antibodies to hidden light chain epitopes) and $\mathrm{N}$ Latex (mixture of monoclonal antibodies to light chain epitopes).

Results: The FLC assay obtained results out of the reference range in 55 cases (52.88\%) with N Latex, and in 63 (60.58\%) with Freelite.

Differences $\geq 105 \%$ (including $11 \%$ of the mean of the difference of the reference intervals $\lambda$ and $K / \lambda$ of both reagents) were observed in 26 cases (25\%) for $\lambda$, and 34 (32.69\%) for $\mathrm{K} / \lambda$.

Involved / uninvolved FLC ratio $\geq 100$ in 4 cases with N Latex and 8 with Freelite, all of them with a known MM diagnosis.

The dispersion diagrams obtained with Deming regression $(K, \lambda$ and $K / \lambda)$ and PassingBablok ( $K$ and $\lambda$ ) presented correlation coefficients (R): $K 0.99 ; \lambda 0.88$ and $K / \lambda 0.92$. The concordance analysis in the Bland-Altman graphs does not indicate agreement for the $\lambda$ measurements. If the Normal distribution for $\lambda$ was rejected, non-parametric tests were performed, and there was evidence of significant differences in the medians matching.

Conclusions: Considering IMGW diagnostic criteria and applying equivalence criteria recommended by CLSI guidelines (Clinical and Laboratorial Standards Institute), the present study shows significant differences with a correlation coefficient $R^{2}<0.95$, reason why we conclude that both assays are not comparable. 
P39

LABORATORIAL STUDY OF ANAEMIA IN ELDERLY NURSERY HOME RESIDENTS- A REALITY OF A DISTRICT HOSPITAL

\author{
$\underline{\text { Ramona-Diana Bindean }}{ }^{1}$, Paula Pinto ${ }^{1}$, Luís Siopa ${ }^{2}$ \\ Clinical Pathology Service ${ }^{1}$ and Internal Medicine Service ${ }^{2}$ of District Hospital of Santarém
}

Introduction: Anaemia is considered a public health problem, being estimated a prevalence of $20 \%$ in the Portuguese population (AWGP). This condition can bring negative outcomes, especially for elderly people, above 65 years old, like higher morbidity and mortality and lower quality of life.

Purpose: The present study pretends to reflect the type and laboratory characteristics of anaemia in elderly population from nursery homes residents.

Materials and Methods: There were consulted individual medical records from January to March of 2018, from nursery home resident older than 65 years of age, who were attended in the emergency service and admitted to an internal medicine service of Hospital District of Santarem. The collected laboratory data in the population with anaemia (haemoglobin concentration $<12 \mathrm{~g} / \mathrm{dL}$ in women and $<13$ $\mathrm{g} / \mathrm{dL}$ in men) include: Haemoglobin, Red blood Cell count, Haematocrit, M.C.V., M.C.H., M.C.H.C., R.D.W., Reticulocyte count, Iron serum, Ferritin, Transferrin saturation, Total iron-binding capacity, Serum vitamin B12, Serum folate, Protein C reactive.

The data was obtained by consulting the individual medical records of the emergency service and completed with the follow-up, if it was the case.

The data was processed and analysed in Excel.

Results: In the study period were attended 256 dwelling-people, from whom only 124 met all the criteria of the study, representing a percentage of $48 \%$.

Anaemia was more common in women than in men with (61\% vs $39 \%$ ). In $64 \%$ of the cases the haemoglobin concentration was superior of $10 \mathrm{~g} / \mathrm{dL}$ in both sexes. In $37 \%$ of the cases the haemoglobin concentration was inferior of $10 \mathrm{~g} / \mathrm{dL}$ and only $3 \%$ have a haemoglobin concentration lower that $7 \mathrm{~g} / \mathrm{dL}$.

The macrocytic anaemia was encountered in $5 \%$ of the group, with values of M.C.V. higher than $101 \mathrm{fL}$. The range of age was 90 years old or older, with decompensated chronic diseases. No relevance difference between sexes. 
The hypochromic, microcytic anaemia was found in $27 \%$ of the studied group, with deficiency of serum iron lower than $50 \mu \mathrm{g} / \mathrm{dL}$, with M.C.V. lower than $83 \mathrm{fL}$ in a percentage of $23 \%$ and $18 \%$ with M.C.H. lower than $27 \mathrm{pg}$. Women are the most affected frequently after 80 years old. The disease they are dwelling is decompensated chronic disease.

In $72 \%$ we encountered normocytic, normochromic anaemia. In the cases of followup, we found that mostly have serum iron deficiency, and in fewer number deficiency of serum vitamin B12 or serum folate. In this group we can find that the episodes in the emergency service where acute (respiratory insufficiency, pneumonia, urinary tract infection). More prevalence in women.

One-third of the group died, mostly with ages superior of 80 years old.

Comments: From the data of this study we can conclude that most of the dwellingpeople suffer of normocytic, normochromic anaemia, with higher prevalence in women.

One of the limitations of the study is in many cases the unknown of the debut of anaemia and rarely do we have information of a rigorous follow-up.

Of the data that we considered important from a laboratorial point of view to characterize anaemia, we can't gathered all of them because of the lack of clinician's orders.

Clinical Pathology has an important role in the diagnosis, study and follow-up of people with anaemia. 
P40

CASE REPORT: “AN OBSCURE AGENT”, PNEUMONIA/ACUTE RESPIRATORY DISTRESS SYNDROME BY VIBRIO ALGINOLYTICUS

$\underline{\text { Isabel Alves Pereira }}{ }^{a}, G^{2}$ Ģa Teles de Abreu ${ }^{a}$, Cristina Pina ${ }^{b}$, Cláudia Barão Ferreira ${ }^{a}$, Rui Ferreira ${ }^{b}$, Rosa Fula Bento ${ }^{a}$, Maria Glória Campello ${ }^{\text {b }}$

a Unidade Local de Saúde do Baixo Alentejo - Hospital José Joaquim Fernandes, Beja

${ }^{b}$ Centro Hospitalar e Universitário do Algarve - Hospital de Portimão

Introduction: Drowning is a serious worldwide threat it affects 30.000 person years in Europe. In 2018, 117 deaths occurred but unlike the WHO report Portugal's drownings are mainly in the age group $>40$ years old; males with alcohol consumption are at highest risk.

Drowning is the process of respiratory injury resulting from immersion or submersion in a liquid, it can lead to atypical infections by aspiration. After initial therapy, secondary deterioration may occur due to lung damage known as acute respiratory distress syndrome that affects the prognosis along with septic shock.

The genus Vibrio has many identified species, the most frequently isolated are cholerae, parahaemolyticus, vulnificus and alginolyticus. Vibrio spp. from the Vibrionaceae family are Gram-negative bacteria, rod-shaped, often curved with mobile flagella. V. alginolyticus natural reservoirs are marine and estuarine waters. Vibrios salt resistance is due to its halophilic character and is more pronounced in V. alginolyticus than in V. cholerae.

V. alginolyticus variants are described as potential reservoirs for virulence genes known in other Vibrio spp. which explains wound, enteric infections and sepsis in humans exposed to seawater.

Our aim is to alert to the value and interpret of laboratory (lab) isolation of an unexpected infectious agent.

Case Report: A 50 year old man is admitted to the Intensive Care Unit with acute pulmonary edema after a drowning event at sea. Fever appears and septic screening and bronchoalveolar lavage (BAL) samples are taken and amoxicillin with clavulanic acid is initiated empirically. At the $4^{\text {th }}$ day the patient maintains fever and the inflammatory parameters keep rising, the isolation result in BAL is positive for methicillin-sensitive S. aureus and V. alginolyticus. Immediate directed antibiotic therapy - cotrimoxazole was given and the clinical evolution was favorable with significant improvement.

Discussion: The authors intend to show that lung infection after drowning can be caused by V. alginolyticus although it has not been described in the literature as a cause of respiratory infection. We demonstrate the importance of clinical history together with lab results to interpret the isolation of this agent and "bring to light" the diagnosis of this patient.

There are no conflicts of interest in all authors. 
P41

CORRELATION BETWEEN THE GLOBULIN CALCULATED INDEX AND THE IMMUNOGLOBULINS CONCENTRATION: A RETROSPECTIVE STUDY (2015-2018)

\author{
$\underline{\text { Luís Marques da Silva }}{ }^{1}$, Vanessa Pereira $^{1}$, Jorge Meneses ${ }^{1}$, Agostinho Lira ${ }^{1}$ \\ ${ }^{1}$ Centro Hospitalar Vila Nova de Gaia/Espinho - Serviço de Patologia Clínica
}

Introduction: The calculated globulin index (CGI), calculated through the formula: total protein - albumin, is one of the most requested laboratory test, mainly for the evaluation of the liver function. However, its value for the detection of immunoglobulin deficits is frequently overlooked.

Goal: To elaborate a retrospective study assessing the correlation between CGI and the immunoglobulins serum concentration and its value in predicting hypogammaglobulinemia.

Materials and Methods: Sample information was extracted from the software system, gathering the period between January 2015 and December 2018, in which total serum protein, albumin and serum immunoglobulins (IgG, IgA e IgM) levels were simultaneously determined.

Analysis was performed using the Biuret method for total protein, colorimetric bromocresol green method for albumin, and nephelometry for immunoglobulin levels.

CGI of each sample was determined and a Passing-Bablok regression and a BlandAltman plot were used to study the correlation between CGI and the sum of the serum immunoglobulins.

The clinical sensitivity and specificity of different CGI cut-offs for suspecting hypogammaglobulinemia was determined as well as its predictive values.

Results: The collected results accounted for a total of 5322 patients, 2491 were male (55 +/- 23 years) and 2831 female $(54+/-22$ years). The correlation coefficient between $\mathrm{CGI}$ and the sum of the serum immunoglobulins was 0.9. Different decision limits were tested. Among the different analyzed cut-offs, a CGI $<2.0$ showed a specificity above $95 \%$ for the detection of a $\operatorname{lgG}<700 \mathrm{mg} / \mathrm{dL}$.

Conclusion: The CGI allows, in an accessible way, to increase the clinical value of two commonly requested assays. In the presence of a low index $(<2.0)$, its high specificity should raise the suspicion of primary or secondary hypogammaglobulinemia. In these cases, sequential investigation with serum protein electrophoresis, immunoglobulin levels and protein immunofixation might be proven useful. 
P42

ADULT COMMON VARIABLE IMMUNODEFICIENCY (CVID): A CASE REPORT

\author{
Maria Helena Rodrigues
}

Centro Hospitalar Póvoa de Varzim/Vila do Conde

Introduction: Common variable immunodeficiency (CVID) is a primary immunodeficiency disorder that results from failed B-cell differentiation with defective immunoglobulin secretion. It has a wide range of clinical presentations: recurrent infections, chronic lung disease, gastrointestinal disorders, autoimmune disorders and an increased susceptibility to lymphoma. It has an estimated prevalence as many as 1 in 25,000. Studies in the United Sates show that CVID is diagnosed in the majority of patients between ages of 20 and 45 . Its clinical heterogeneity may explain the delayed diagnosis after the onset of symptoms.

Clinical presentation: A 40-year-old male presented with lipothymia and a one-day history of fever, asthenia and headache, without complaints of chest pain, palpitations, cough or dyspnea. On admission his vitals were as follows: temperature $=38,5^{\circ} \mathrm{C}$, heart rate $=92$ beats $/$ minute, blood pressure $=100 / 67 \mathrm{mmHg}$, eupneic, and $\mathrm{SpO}_{2}=95 \%$ in room air. Auscultation revealed normal breath sound. Laboratory study demonstrated leukocytosis $\left(28,200 / \mathrm{mm}^{3}\right)$ with neutrophilia $(92,5 \%)$ and C-reactive protein $=7,29 \mathrm{mg} / \mathrm{dL}$ (normal $<0,5$ ). Chest radiograph revealed opacities in the inferior zones of the left lung. High resolution computed tomography chest scan confirmed the suspicion of an infectious etiology. The patient was diagnosed with community acquired pneumonia and started on amoxicillin clavulanic acid and azithromycin. Routine laboratory workup revealed significantly reduced levels of all immunoglobulins (IgG $=101 \mathrm{mg} / \mathrm{dL}$, IgA $<5 \mathrm{mg} / \mathrm{dL}$, IgM $=8 \mathrm{mg} / \mathrm{dL}$ ). There was a past medical history of recurrent rhinosinusitis since childhood and an admission to an intensive care unit for pneumonia. In view of this information, the clinical pathologist reported the results to the clinician and suggested additional laboratory studies. Flow cytometry of leukocytes disclosed B lymphopenia, reduced levels of switched memory $B$ cells, T lymphopenia and T cell impaired function. Inadequate antibody response to pneumococcal vaccine, tetanus and diphtheria toxoid confirmed the diagnosis of CVID.

Conclusion: This case highlights the importance of cooperation between the laboratory and clinicians on establishing an uncommon pathology, whose outcome greatly improves with early diagnosis. 
P43

TUMOR MARKERS IN THE PLEURAL FLUID: WHAT'S THE VALUE?

Maria Manuela Rebordão; Sandra Francisco; Ana Luísa Vieira; Manuel Silva Serviço Patologia Clínica, Hospital Forças Armadas, Lisboa, Portugal

Pleural effusion is a frequent cause of hospital admission. About $5-20 \%$ of pleural effusions have a neoplastic etiology and may be the first sign of clinical presentation. The use of tumor markers in the differential diagnosis of pleural fluid is not consensual. There are studies that indicate that the tumor markers fluid /serum (F/S) ratio $>1,2$ is important to the diagnosis of neoplastic effusion.

Goal: To study tumor markers in the pleural fluid, to calculate the $\mathrm{F} / \mathrm{S}$ ratio and to determine its importance in the clinical diagnosis.

Methodology: Effusion fluid and serum samples were collected and analyzed on the same day. CEA, CA15.3 and Ca19.9 were determined using a chemiluminescent method (Architect - Abbott Diagnostics) and F/S ratio were calculated. The reference values in serum were also applied for determination of the tumor markers in pleural fluid.

Population: We studied all the pleural fluids addressed to our laboratory in the last year for a total of 15 patients. All had an etiological diagnosis to clarify. Average age was 67,6 years, 3 females and 12 males.

Results: We divided the population in 2 groups: Group-1, Negative pleural fluid for tumor markers and a F/S ratio < 1,2 - 9 patients: 7 without oncologic pathology; 2 with lung cancer, diagnosed by bronchial brushing and pulmonary biopsy.

Group-2, One or more positive tumor markers and a F/S ratio $>1,2-6$ patients: 2 with prior diagnosis of lung cancer: One with F/S CEA - 9,81 and F/S CA19.9 - 30,3 and another with $\mathrm{F} / \mathrm{S}$ CA15.3 - 1,43 with very high values of CA19.9 in serum alone; 2 patients with metastatic endometrial adenocarcinoma and a F/S ratio CA15.3 of 6,3 and 6,2. A patient with a hemato-lymphoid lung cancer (autopsy) and F/S CEA of 202,2; 1 patient with poorly differentiated lung adenocarcinoma (lung biopsy) - the values for all the markers were high and $\mathrm{F} / \mathrm{S}$ ratios were CEA-20,1, CA19.9-2,55 and CA15.3- 2,55.

Conclusion: Determining tumor markers and finding out the $\mathrm{F} / \mathrm{S}$ ratio $>1,2$ in pleural fluid allowed to identify a neoplastic pleural effusion in $100 \%$ of the studied cases. In 2 patients the determination of tumor markers in pleural fluid with a $F / S$ ratio $<1,2$ was not compatible with neoplastic diagnosis leading to false negatives. Thus, tumor markers and $\mathrm{F} / \mathrm{S}$ ratio may take an important value in elucidating the etiology of pleural effusion. 
P44

DIAGNOSTIC UTILITY OF HIGH SENSITIVITY CARDIAC TROPONIN I IN THE DIAGNOSIS OF NON-ST-ELEVATION MYOCARDIAL INFARCTION (NSTEMI): CASE REPORT

\author{
A.Paradela, E.Figueiredo, E. Ramalheira \\ Serviço Patologia Clínica, Centro HospitalarBaixo Vouga-EPE
}

Background: High-sensitivity cardiac Troponin I (hs-cTnl) is a cardiac biomarker of choice in patients suspected with acute coronary syndrome (ACS). The international federation of clinical chemistry recommends the term high-sensitivity to be reserved for an assay meeting two criteria: the coefficient of variation must be $<10 \%$ at the 99\% percentile, and concentrations should be measurable (above the limit of detection) in at least $50 \%$ of healthy reference population. With the ability to detect small increases in circulating, high-sensitivity assays have substantially increases the diagnostic sensitivity for early detection of acute myocardial infarction. However, evolution has led to a decrease in diagnostic specificity, diagnostics algorithms based in cardiac troponin over time $(\Delta)$ have shown the ability to improve diagnostic specificity into clinical practice.

Case report: A 57 years old man presented on the emergency room with several hours of chest pain associated with nausea. He stated that the pain radiated to his right arm. He denied dyspnea and vomiting. He had a medical history of hypertension and dyslipidemia. The patient ECG showed absence of changes. Blood was sampled for hscTnl (Siemens ADVIA-Centaur hs-cTnl assay) on presentation and the concentration was elevated to $5591 \mathrm{pg} / \mathrm{ml}$. Three hours later hs-cTnl concentration was $10318 \mathrm{pg} / \mathrm{ml}$ (delta=4727). Patient was transferred to the Cardiac Catherization Unity where angiography was performed and revealed obtuse marginal branch circunflex artery occluded in the beginning and right coronary artery with diffuse irregularities and $30 \%$ stenosis in the middle third.

Conclusion: As seen in our case, high sensitivity Troponin I plays a vital role in the diagnosis of acute coronary syndrome in the absence of changes in diagnostic electrocardiogram. A normal ECG does not rule out myocardial ischemia/infarction. An algorithm incorporating hs-cTnl concentration and delta cTnl values accurate diagnosis of NSTEMI. 


\section{P45}

THERAPEUTIC MONITORING OF PLATELET AGREGGATION: REGARDING 2 CLINICAL CASES

Teresa Pina, Anália do Carmo, João Pego, Fernando Rodrigues

Serviço de Patologia Clínica - Centro Hospitalar e Universitário de Coimbra

Coimbra, Portugal

Introduction: Platelet activation and aggregation play a key role in atherothrombotic disease. The use of antiplatelet therapy such as aspirin, and/or adenosine diphosphate receptor inhibitors (clopidogrel or prasugrel) may prevent arterial thrombotic complications. Interindividual variability in the response to antiplatelet drugs, can put some patients at increased risk of recurrent events.

The authors present two case reports that emphasize the role of electrical impedance aggregometry (EIA) in antiplatelets drug monitoring.

Methods: Platelet aggregation was evaluated by EIA according the manufacturer recommendations (Multiplate ${ }^{\circledR}$ ). EIA measures the increase in impedance upon the addition of platelet agonists such as adenosine diphosphate, collagen, ristocetin, thrombin receptor activating peptide, and arachidonic acid.

\section{Results:}

Case report 1: A 55-years-old female patient with history of ischemic stroke of the middle cerebral artery in 2012, medicated since then with acetylsalicylic acid (ASA) $100 \mathrm{mg} 1 \mathrm{id}$ and without other ischemic events. EIA showed a significant reduction of aggregation in response to the arachidonic acid (10U for a reference interval of $71 \mathrm{U}$ $115 \mathrm{U})$

Case report 2: A 39-years-old male patient, with history of ischemic stroke in the posterior inferior cerebellar artery territory in 2012 and a transient ischemic attack in January 2019 , currently medicated with clopidogrel $75 \mathrm{mg}$, 1id. No changes in platelet aggregation were observed with any of the agonists.

Discussion: The first patient showed a marked reduction of platelet aggregation with arachidonic acid which is in accordance with the intake of ASA. The second patient, in spite of the clopidogrel prescription showed no alteration in the platelet aggregation. This result may indicate nonadherence/noncompliance, variability of absorption or metabolization, presence of genetic polymorphisms and/or environmental factors. Furthermore, this result could be correlate with the transient ischemic attack in January 2019 and emphasizes the need to change the antiplatelet drug. These two case report showed that the evaluation of platelet inhibition by EIA could be a suitable option to improve clinical outcome of patients that are under antiplatelet therapy. 
P46

INVASIVE FUNGAL INFECTION OF SPHENOID SINUS - A HEADACHE FOR BOTH THE PATIENT AND THE PHYSICIAN

Zuzana Melník ', Hugo Cruz', Virgínia Lopes', Graça Henriques², Cristina Macedo Freitas $^{3}$, Helena Ramos ${ }^{1}$

${ }^{1}$ Microbiology Service, Department of Pathology, CH Universitário do Porto, Portugal ${ }^{2}$ Centralized Laboratory (CoreLab), Department of Pathology, $\mathrm{CH}$ Universitário do Porto, Portugal

${ }^{3}$ Nephrology Service, Department of Medicine, $\mathrm{CH}$ Universitário do Porto, Portugal

Background: Fungal sinusitis is a fungal infiltration in sinus mucosa and may vary from localized to life-threatening invasive infection. Aspergillus spp. and Mucorales are important agents causing the most serious infections, particularly in the setting of immunosuppression (therapy for haematologic malignancies, haematopoietic cell or solid organ transplantation). Sphenoid sinus fungal infection is rare, but it is more aggressive than invasive fungal infection of the other paranasal sinuses.

Case Report: A 49-year-old women with a history of kidney transplantation and posttransplant diabetes mellitus presented with a 4-month left side headache associated with significant malaise and weight loss. The pain, initially localized in the left frontal and temporal regions, later on progressed to both medial canthus of the eyes. Computed tomography (CT) scans showed wall thickening of sphenoid sinus, sphenoid opacification and heterogeneous density content with small central calcification and bone destruction of both sphenoid septum and sela turca, which were highly suggestive of chronic, locally invasive fungal infection. The patient was submitted to endoscopic sinus surgery with debridement and bilateral sphenoidotomy. Greyishwhite dense sphenoid sinus content was sent for histopathology and microbiology analysis.

Results: Histopathology analysis revealed extensive tissue necrosis and numerous fungal hyphae. As microbiology study identified Aspergillus spp.the patient started treatment with voriconazol for a period of 8-weeks. Follow-up consultations showed complete resolution of clinical symptoms and remission of invasive fungal sinusitis.

Conclusions: Although rare, invasive fungal sinusitis must be considered when facing adequate clinical symptoms and risk factors. Compatible CT images combined with tissue invasion on histopathology and microbiology analysis provide definite diagnosis of invasive fungal infection. Effective treatment relies on proper surgical debridement and specific anti-fungal therapy based on isolation and identification of the infecting fungus. A delay in diagnosis and treatment may result in serious complications. 
P47

ACUTE ENDOCARDITIS BY HAEMOPHILUS PARAINFLUENZAE: AN UNEXPECTED DIAGNOSIS

Adão $L^{1}$, Muzyka $V^{1}$, Lemos $C^{1}$, Carneiro $L^{1}$, Leitão $I^{2}$, Pires $D^{2}$, Marques $T^{2}$, Santos $C$ $\mathrm{M}^{2}$, Lito $\mathrm{LM}^{1}$, Cristino $\mathrm{J} \mathrm{M}^{1}$, Caldeira $\mathrm{L}^{2}$

${ }^{1}$ Clinical Pathology Service CHULN (Centro Hospitalar Universitário Lisboa Norte)

${ }^{2}$ Infeccious Diseases Service, CHULN

Introduction: HACEK group are fastidious Gram negative microorganisms. that typically colonize oropharynx. They are responsible for $3 \%$ of acute endocarditis $(A E)$ of native valve. We report a case of AE by $H$. parainfluenzae.

Case Description: A 27-year-old melanodermic man, from São Tomé, living in Portugal for 12 years, was admitted in Emergency Service on October $8^{\text {th }}, 2018$ with fever, up to 40 으, with 48 hours of onset, chills, myalgias and frontal headaches and was stable. He had history of many non-treated tonsillitis episodes in childhood. In the physical exam he had no relevant alterations. He had neck stiffness, without focal neurological deficits. Laboratory tests revealed neutrophilia, (with no leucocytosis), thrombocytopenia (77 000/Ul), RCP $46.3 \mathrm{mg} / \mathrm{dl}$, with no renal or liver function Impairment. Urinalysis: proteinuria $(150 \mathrm{mg} / \mathrm{dl})$. The electrocardiogram showed sinusal tachycardia. Lumbar punction, chest radiograph and cranial CT scan didn't show any findings. Abdominal Ultrasound (US) revealed a homogeneous splenomegaly $(12 \mathrm{~cm})$. The patient was admitted in Infectious Diseases Service with a provisory diagnosis of Mediterranean Spotted Fever (MSF) and he was started with doxycycline. The serology for HIV, MSF, Q fever and leptospirosis, as TPHA test were negative. Two sets of blood cultures grew multissensitive $H$. parainfluenzae. Transoesophageal echocardiogram (TE) showed "thickening of the distal 1/3 of both leaflets of the Mitral Valve (MV) with two hypermobile vegetations and moderate regurgitation, aortic valve (AV) was thickened on the tip of the left coronary cuspid, suggesting endocardial involvement". It was made the $H$. parainfluenzae AE diagnosis. The patient started ampicillin and gentamicin, for 28 days, according to AE treatment Guidelines. After lowering of inflammatory markers, the patient underwent TE which showed no AV vegetations. or thickening and a decrease of the MV vegetations.

Conclusion: HACEK group is a rare cause of AE. Though $\mathrm{H}$. parainfluenzae is usually thought of low pathogenicity, endocarditis caused by these bacteria, typically presents sub-acutely, with vegetations and a high risk of septic embolization.

Blood cultures are crucial to identify this agent. According to anamnesis and US, we consider the hypothesis of an underlying rheumathic heart disease. 
P48

PREVALENCE OF ANEMIA IN 2018

Isabel Cachapuz $^{1}$

${ }^{1}$ Unidade Local de Saúde de Matosinhos

Introduction: In Portugal, the prevalence rate of anemia is higher than the average of the developed countries, since in these countries the rate is $9 \%$, the national rate is more than double, reaching $20 \%$. The situation of this is an alarming disease. At the national level, this problem has as its roots the underdiagnosis, lack of knowledge and, consequently, under-treatment, with negative impact on the portuguese population.

Objectives: To assess prevalence of anemia in hospitalized patients who use the Urgency Service (SU) of a Northern Hospital in 2018, in adults only.

Material and Methods: Retrospective study. Adult population ( $>=18$ years). Inclusion Criteria: Hemoglobin in the male case $<13.6 \mathrm{~g} / \mathrm{dL}$ and female $<11.5 \mathrm{~g} / \mathrm{dL}$. Results: During the year 2018, 86242 patients were recruited and 14485 were hospitalized. All adults. A) Regarding SU, 9305 had anemia, 59\% were male and 41\% were female; The mean hemoglobin value is $11.05 \mathrm{~g} / \mathrm{dL}$, a minimum value of $3 \mathrm{~g} / \mathrm{dL}$ and a maximum value of $13.5 \mathrm{~g} / \mathrm{dL}$. Average age of 70 years. B) Regarding hospitalization, 4756 had anemia, $53 \%$ were male and $47 \%$ were female; The mean hemoglobin value is 10.52 $\mathrm{g} / \mathrm{dL}$, a minimum value of $3.8 \mathrm{~g} / \mathrm{dL}$ and a maximum value of $13.5 \mathrm{~g} / \mathrm{dL}$. Average age of 68 years. The Anemia Prevalence in adults who use the SU is $11 \%$ and in hospitalized adults it is $33 \%$.

Conclusion: This study showed that the prevalence of Anemia is high both in the SU and among hospitalized patients. It was not possible, in this study, to characterize the type of anemia, nor the causes of it, many times multifactorial. Although anemia affects approximately one in five portuguese, the perception of disease is very low, suggesting a high rate of undiagnosed / untreated cases, and it is therefore urgent to develop a plan to improve the knowledge of this situation, especially in the larger groups. risk. It is urgent to develop strategies aimed at increasing the perception, screening, diagnosis and treatment of anemia cases in the portuguese population. Increased recognition of anemia will play a crucial role in reducing the impact of this public health problema. 
P49

SWEAT CHLORIDE TEST IN THE DIAGNOSIS OF CYSTIC FIBROSIS: RECOMMENDATIONS AND LIMITATIONS.

\author{
Lília Figueira1, Mariana Vaz ${ }^{1}$; Helena Proença ${ }^{1}$ \\ ${ }^{1}$ Centro Hospitalar Universitário de Lisboa Norte, EPE
}

Background: Cystic Fibrosis (CF) is a severe, multisystem, inherited condition. A complete diagnostic evaluation should include a newborn screening, a sweat chloride test, a genetic test, and a clinical evaluation at a referral care center.

The quantitative sweat test is the current gold standard, providing confirmation of the diagnosis in approximately $98 \%$ of the CF cases.

This test is a composite of three separate, sequential procedures that must be accomplished without interventional error, by specialized laboratory professionals. In order, the steps are (1) sweat stimulation by pilocarpine iontophoresis, (2) sweat collection with the Macroduct ${ }^{\circledR}$ disposable sweat collector, and (3) sweat electrolyte analysis.

It doesn't require any preparation, however there are some technical recommendations and pathophysiological conditions associated with false results.

Aim: The aim of this review is to examine the role of the sweat test and its limitations in the diagnosis of CF.

Methods: This work is based in our hospital experience with sweat tests, as a referral center and in a review of the literature.

Results: Many conditions can interfere with the sweat electrolyte analysis, from the sweating physiology to the rigors of a properly performed test and its interpretation. Some of the most common situations include physiological variations, oedema, the use of mineralocorticoids, untreated Addison's disease, ectodermal dysplasia, some types of glycogen storage diseases, untreated hypothyroidism, as well as insufficient sweating due to several factors such as age, skin condition, hydration status and collection system.

Even if carried out by an experienced technician, technical errors can occur, such as evaporation and contamination.

Furthermore, the sweat test must be performed at least twice in patients with positive or borderline results, preferably several weeks apart, as well as in patients with a strong clinical suspicion or who don't follow a typical course of CF.

Conclusions: With this review it is possible to understand the relevance of the sweat test and how important it is to be performed by a trained technician in an experienced and reliable laboratory, in order to assure the quality of the results. 
P50

CALIaGold $^{\circledR}$ and BÜHLMANN fCAL $^{T M}$ TURBO: A COMPARISON OF TWO COMMERCIAL
IMMUNOASSAYS FOR THE QUANTIFICATION OF FAECAL CALPROTECTIN

Figueira, ${ }^{1}{ }^{1}$, Coiana, M. ${ }^{1}$; Silva, B.M.P. ${ }^{1}$; Couto, S.P.S.G. ${ }^{1}$; Carriço Da Silva, M.S. ${ }^{1}$; Proença, H. ${ }^{1}$

${ }^{1}$ Centro Hospitalar Universitário de Lisboa Norte, EPE

Background: Calprotectin, a calcium-binding protein produced in cells such as neutrophils, is released during inflammation

Faecal calprotectin ( $\mathrm{fCal}$ ) is a biomarker used to distinguish between inflammatory bowel disease (IBD) and non-inflammatory bowel conditions, and it is strongly correlated with histological and endoscopic parameters of IBD activity.

Both CALiaGold ${ }^{\circledR}$ and BÜHLMANN fCAL ${ }^{\text {TM }}$ turbo analyzers are particle enhanced turbidimetric immunoassays (PETIA) capable of measuring fCal levels.

Aim: The aim of this study was to compare the performance of a new automated assay for quantitative results of fCal, CALiaGold ${ }^{\circledR}$ test on SENTiFIT ${ }^{\circledR} 270$ analyzer (Sentinel ${ }^{\circledR}$ ) versus our routine method, BÜHLMANN fCAL ${ }^{\mathrm{TM}}$ turbo on Cobas $^{\circledR} 8000$ analyzer (Roche ${ }^{\circledR}$ Diagnostics).

Methods: The study was performed between Aug 14th and Nov 12th, 2018. 315 routine faecal samples extraction was carried out using the CALiaGold ${ }^{\circledR}$ Tube and CALEX ${ }^{\circledR}$ Cap devices.

The data was analyzed with NCSS16 statistics software. Concordance and correlation analysis between methods were carried out via Spearman correlation, contingency table and Robust Linear Regression (Passing-Bablok Median). For concordance analysis, the manufacturer's cut-off ( $\geq 50 \mu \mathrm{g} / \mathrm{g}$ ) was used.

Results: The Spearman correlation coefficient between both methods was 0. 957, reflecting a very strong positive correlation. Similarly, the concordance was $93,97 \%$ showing a substantial agreement between methods. Using $\mathrm{fCAL}^{\mathrm{TM}}$ turbo as comparison method, CALiaGold ${ }^{\circledR}$ showed $95.0 \%$ of sensitivity and $90.5 \%$ of specificity. The negative predictive value and positive predictive value were $84.8 \%$ and $97.0 \%$, respectively.

Conclusions: The symptoms of intestinal disorders are relatively non-specific. It is important and profitable to distinguish between patients with functional diseases, which do not require invasive methods, and those with organic disorders - particularly IBD.

The CALiaGold ${ }^{\circledR}$ assay on SENTiFIT ${ }^{\circledR} 270$ analyzer showed a good performance versus $\mathrm{fCAL}^{\mathrm{TM}}$ turbo. Therefore, the CALiaGold ${ }^{\circledR}$ assay is judged to be eligible for the measurement of calprotectin in routine settings that offer a unique solution to analyze both faecal calprotectin and occult blood on a single equipment (SENTiFIT ${ }^{\circledR} 270$ ). 
P51

B-CELL NON HODGKIN LYMPHOMA: A CASE REPORT ILLUSTRATING THE UTILITY OF PERIPHERAL BLOOD SMEAR REVIEW ON THE DIAGNOSIS OF HEMATOLOGIC MALIGNANCIES

\section{Maria Helena Rodrigues}

\section{Centro Hospitalar Póvoa de Varzim/ Vila do Conde}

Introduction: Non-Hodgkin lymphomas (NHL) are a heterogeneous group of B-cell and T-cell malignancies arising from lymphoid tissue, with diverse clinical and biological features. The clinical presentation of NHL varies widely depending on the type of lymphoma and the areas of involvement.

Case presentation: A 46-year-old male presented to the emergency department (ED) with a one-week history of worsening abdominal discomfort in the left inferior quadrant, after a single episode of vomiting and diarrhea that had improved with symptomatic treatment. Physical examination was inconspicuous, except for a cervical tumefaction. Laboratory study showed anemia (hemoglobin $=12.7 \mathrm{~g} / \mathrm{dL}$ ), thrombocytopenia $\left(110,000 / \mathrm{mm}^{3}\right)$, slight leukocytosis $\left(12,170 / \mathrm{mm}^{3}\right)$ and lymphocytosis (63\%). The complete blood count was executed on Sysmex XE-5000 that gave no morphologic flag. It was repeated on Advia 2120, with similar results, but with a morphologic flag (Blasts++). A blood smear review was performed. It revealed a $38.5 \%$ count of abnormal lymphocytes (small-medium sized pleomorphic cells with scant weakly basophilic cytoplasm; irregular nucleus with immature chromatin pattern, some exhibiting a nucleolus). The results were reported to the ED doctor. Thereafter, the initial hypothetical diagnosis of diverticulitis was reviewed. A more exhaustive anamnesis disclosed a 6-month history of night sweats and increasing asthenia not valued by the patient. He was admitted for further diagnostic workup. Chest-abdominal-pelvic computed tomography scan showed disseminated lymphadenopathies and hepatosplenomegaly. Flow cytometry study of the peripheral blood lymphocytes suggested the diagnosis of a B-cell NHL (mantle cell lymphoma vs marginal zone B-cell lymphoma/lymphoplasmacytic lymphoma).

Conclusion: Technological improvements in the performance of hematology analyzers have decreased the requirement for microscopic smear review and manual differential count. However it still remains an important diagnostic tool, and consensus guidelines have been established for when such review should be performed. This case emphasizes the usefulness of manual peripheral blood smear review in the suggestion of a differential diagnosis and also in triggering recommendations for patient further evaluation. 
P52

INFLUENZA - EPIDEMIOLOGICAL STUDY OF A CENTRAL HOSPITAL

Cláudia Pereira, Lurdes Correia, Lucília Araujo, Vanda Mota, Henriqueta Pereira, Fernando Rodrigues

Serviço de Patologia Clínica do Centro Hospitalar e Universitário de Coimbra

Introduction: Influenza is an acute, contagious respiratory disease caused by viruses. Transmission is by respiratory route. Viruses are Influenza A (InfA) and Influenza B (InfB). Symptoms include fever, chills, cough, nasal congestion, headache, and myalgia.

Aim: Assess the epidemiology of influenza in the adult population ( $\geq 18$ years) in a central hospital in two consecutive seasons (week 40/2016 - 20/2017 and week 40/2017 - 20/2018).

Material and methods: Data were collected from Clinidata $X X I^{\circledR}$ software. Detection of influenza viruses was performed by real-time PCR technology on GeneXpert ${ }^{\circledR}$, in nasopharyngeal swabs.

Results and conclusions: In the $2016 / 2017$ season, 732 samples were analyzed. InfA was detected in 307, and InfB wasn't detected. The number of samples from male and female was the same $(n=366)$. InfA was detected mostly in males ( $n=161)$. The majority of samples came from the emergency department (ED) ( $n=448$ ), hospitalization ( $n=187$ ) and intensive care units (ICU) $(n=51)$. In samples with InfA, the distribution was similar with higher number in ED ( $n=200)$, hospitalization $(n=$ 73 ) and ICU $(n=14)$. The median age of the patients with virus detection was 71 years. The period of incidence was from week 46/2016 to 6/2017, with peak from week $50 / 2016$ to $2 / 2017$. In the $2017 / 2018$ season, there was an increase in the number of samples ( $n=1982$ ), with detection of InfA in 228, InfB in 554 and the two viruses simultaneously in 3 samples. The number of female samples was higher $(n=1112)$. ED was the highest number of requests ( $n=1592)$, followed by hospitalization $(n=258)$ and ICU ( $n=78$ ). InfA was detected mostly in female individuals ( $n=134$ ) and the median age was 61.5 years. Most samples were from ED $(n=200)$. The majority of detections occurred from week $52 / 2017$ to $16 / 2018$, with peak from week 6/2018 to $10 / 2018$. In InfB, most of the detections were also in female $(n=339)$ and median age was 70 years. The ED has the highest number of detections $(n=477)$. The majority of detections was from week $51 / 2017$ to $12 / 2018$ with the peak between week $2 / 2018$ and $9 / 2018$. The 3 cases with InfA and InfB simultaneously belonged to female individuals from the ED $(n=1)$ and hospitalization $(n=2)$ and occurred in the peak period.

These results are in line with those reported by the National Influenza Surveillance Program for viruses detected and period of detection. 
P53

\section{COLORECTAL CANCER SCREENING - ONE YEAR EXPERIENCE}

Cláudia Pereira, Ângela Silva, Ana Cristina Marques, Fernanda Escada Fontes, Fernando Rodrigues

Serviço de Patologia Clínica do Centro Hospitalar e Universitário de Coimbra

Introduction: Colorectal cancer (CRC) is a major cause of mortality and morbidity worldwide. Screening programs can have an impact in reduction in incidence and mortality. In accordance to the European recommendations, the Portuguese Directorate-General for Health considers screening for CRC a necessity. It is recommended to perform a primary test with faecal occult blood test (FOBT) on the asymptomatic population from 50 to 74 years old, without risk factors. Patients with a positive FOBT should then proceed to colonoscopy.

Our Hospital's laboratory is, since December 2017, responsible for the processing of FOBT samples from its geographical area. Samples are analysed with HM-JACKarc (A. Menarini Diagnostics), using antibodies to detect haemoglobin. Samples are considered positive when faecal haemoglobin $(\mathrm{f}-\mathrm{Hb})$ is equal or greater $(\geq)$ than 15 $\mu \mathrm{g} / \mathrm{g}$.

The aim of our work is to provide a description of the population screened in our region and the results obtained during the year 2018.

Description: A total of 14,707 samples were collected and analysed, $56.8 \%$ from females and $43.2 \%$ from males. The median age was 62 yo (interquartile range [IQR]: 68-56). The minimum age was 49 and the maximum age 76 yo. $1.4 \%$ of the analysed samples were outside the screening's target age. $93.6 \%$ of the analysed samples were negative and $6.4 \%$ positive. The median $\mathrm{f}-\mathrm{Hb}$ concentration detected was $30 \mu \mathrm{g} / \mathrm{g}$ (IQR: 50-13).

In regard to negative screenings, $57.5 \%$ were female and $42.5 \%$ male, with a median age of 62yo (IQR: 68-56) and a median f-Hb concentration of $0.5 \mu \mathrm{g} / \mathrm{g}$ (IQR: 1.2-0.2). Of the positive screenings, $53.2 \%$ were male and $46.8 \%$ female. The median age was 64yo (IQR: 70-58). The median haemoglobin concentration was $40 \mu \mathrm{g} / \mathrm{g}$ (IQR: 97-22).

Discussion: Based on these results, $6.4 \%$ of the patients who performed the screening test had indications for additional studies. As we would expect in a screening test, the percentage of negative tests represents the majority. $1.4 \%$ of participants were outside of the target age.

In regards to the population with positive screening, one can perceive the tendency to a slightly older age and male predominance as in accordance to the disease's known epidemiological profile.

Screening programs and early therapeutic intervention could, in time, substantially reduce the morbidity and mortality associated with CRC. 
P54

WHAT HAPPENS FOLLOWING A POSITIVE COLORECTAL CANCER SCREENING?

Cláudia Pereira, Ângela Silva, Ana Cristina Marques, Fernanda Escada Fontes, Fernando Rodrigues

Serviço de Patologia Clínica do Centro Hospitalar e Universitário de Coimbra

Introduction: Our Hospital's laboratory processes samples for faecal occult blood test (FOBT) as a part of a colorectal cancer (CCR) screening programme. Analysis are performed in HM-JACKarc (A. Menarini Diagnostics), using specific antibodies to detect human haemoglobin $(\mathrm{Hb})$.

Our aim was to assess retrospectively the follow-up of patients with positive FOBT, whether or not they proceed to colonoscopy and its results.

Description: In 2018, $6.4 \%$ of the 14,707 received samples were positive. We studied among these the population of 182 patients who had clinical data accessible through our hospital's information system.

The population's median age ( $n=182$ ) was 64 (interquartile range [IQR]: $70-58$ ). $55.5 \%$ of the samples belonged to males and $44.5 \%$ to females. Data was available for 182 patients, showing that $45.1 \%(n=82)$ had colonoscopy and $33 \%(n=60)$ did not.

Those who underwent colonoscopy, $61 \%$ were males and $39 \%$ females, median age was 64.5 (IQR: 70-59) and median $\mathrm{Hb}$ was $32.2 \mu \mathrm{g} / \mathrm{g}$ (IQR: 122-20). In those who did not, $51.7 \%$ were females and $48.3 \%$ males, median age was 64 (IQR: $70-59$ ) and median $\mathrm{Hb}$ was $30 \mu \mathrm{g} / \mathrm{g}$ (IQR: 90-20). When the result of the colonoscopy was available $(n=75), 12 \%(n=9)$ were normal, $77.3 \%(n=58)$ showed benign abnormalities and $10.7 \%(n=8)$ identified malignant tumours.

Median age was 58 (IQR: 67-55), 65.5 (IQR: 71-59) and 68 (IQR: 72-60), respectively, for patients with normal, benign and malignant results in the colonoscopy. The male:female proportion was 2:1, 1.2:1 and 8:0, respectively, for the same groups. Median $\mathrm{Hb}$ values also showed increasing tendency, with $20 \mu \mathrm{g} / \mathrm{g}$ (IQR: 21-18), $41 \mathrm{ug} / \mathrm{g}$ (IQR: 122-22) and $50 \mu \mathrm{g} / \mathrm{g}$ (IQR: 215-28), respectively.

The dates for sample collection and colonoscopy was known for 76 of the patients, and an average number of 77 days occurred between these two moments.

Discussion: Most individuals participating in the screening accepted to proceed with colonoscopy facing a positive result - revealing mainly benign abnormalities. In comparison, in malignant cases, median age and $\mathrm{Hb}$ concentration was found to be higher. Malignancies were identified only in males, being compatible with the epidemiology of CCR, more prevalent in men. This findings suggest that the FOBT interpretation could be benefit from a risk stratification algorithm, pondering gender, age and $\mathrm{Hb}$ concentration. 
P55

A CASE OF CYSTINURIA IN A YOUNG ADULT

Carla Gonçalves Ferreira, Ezequiel Moreira e Helena Ferreira da Silva

Serviço de Patologia Clínica - Centro Hospitalar Médio Ave, E.P.E.

Introduction: About $10 \%$ of the general population suffers with one of the most common disorders of the urinary tract: kidney stones. In early ages, kidney stones can be due to rare inherited metabolic disorders like cystinuria. This is characterized by impaired reabsorption of cystine, lysine, ornithine and arginine in the proximal tubule with an increased urinary excretion. Patients with cystinuria are more likely to develop chronic kidney disease, as a result of recurrent kidney stones due to the low solubility of cystine in urine. Given the severity of this condition it's important to achieve an early diagnosis in order to implement the appropriate therapeutic. If these diseases are not recognized early, it may lead to a delay in kidney injury prevention.

Case report: A healthy 19 years old male visited our emergency department for the second time in two days complaining of dysuria and hematuria, denying other symptoms like diarrhea, nausea, pain or fever. The patient was diagnosed with urinary tract infection starting treatment with ciprofloxacin at the first urge to the emergency. The blood tests were all normal, without leukocytosis or neutrophilia and C-reactive protein $<0,1 \mathrm{mg} / \mathrm{dL}$. The urinalysis showed $\mathrm{pH} 6.5$, proteins $50 \mathrm{mg} / \mathrm{dL}, 916 \mathrm{red}$ cells/field with no white blood cells. This urinalysis also revealed six-sided crystals, which are pathognomonic of cystinuria. The patient continued the antimicrobial therapy for 4 days more, being referred to nephrology consultation, in order to proceed further studies and surveillance.

Discussion: In management of rare kidney stone disorders, like cystinuria, it's essential to implement a medical therapy promptly able to reduce stone production and to decrease the potential impact on kidney function. So, it's important to recognize this disorder early, helping delay or prevent the development of end stage kidney disease. 
P56

CYTOGENETIC ABNORMALITIES (FISH) MOST FREQUENTLY FOUND IN NON HODGKIN LYMPHOMA

Marisa Ferreira, Naseelah Mussá, Cristina Duarte

Hospital de Santa Maria - CHULN, E.P.E

Introduction: Non Hodgkin Lymphomas (NHL) are clonal tumours of B-cell origin at various stages of differentiation. Morphology and immunophenotype are useful for the diagnosis in B-cell lymphomas. The NHL subtypes vary in their relative frequency in different parts of the world and the median patient age for all types is in the sixth or seventh decade of life, with a male predominance. Genetic features have an increasingly important role in the classification of lymphoid malignancies. Genetic studies like Fluorescence In Situ Hybridization (FISH) are valuable diagnostic tools for the identification of translocations associated with some disease entities. Several NHL have characteristic genetic abnormalities that are important in determining their biological features and can be useful in differential diagnosis, such as: $t(11 ; 14)$ in Mantle Cell Lymphoma, $\mathrm{t}(14 ; 18)$ in Follicular Lymphoma, $\mathrm{t}(8 ; 14)$ in Burkitt Lymphoma and $t(11 ; 18)$ in MALT Lymphoma.

Goals: To describe the most frequent cytogenetic abnormalities in cases of NHL from $01 / 01 / 2016$ to $31 / 12 / 2018$ in a tertiary referral hospital.

\section{Materials and methods:}

Data search by: Clinidata XXI ${ }^{\circledR}$ Software (Maxdata).

FISH probes used in bone marrow blood samples: Vysis ${ }^{\circledR}$ IGH/MYC/CEP 8 Tri-color DF, Vysis ${ }^{\circledR}$ LSI $^{\circledR}$ IGH/BCL2 Dual Color Dual Fusion, Vysis ${ }^{\circledR}$ IGH/CCND1 DF, Vysis BIRC3/MALT1 DF, Vysis ${ }^{\circledR}$ LSI BCL6 Dual Color Break Apart Rearrangement.

Results: From 153 patients with NHL immunophenotypic diagnosis, $47.1 \%$ were females and $52.9 \%$ males. The median of ages was 67.7 (with a minimal age of 27 and maximal of 93). The percentage of $\mathrm{NHL}$ patients with positive results for each of the cytogenetic abnormalities studied were $4.6 \%$ for $\mathrm{t}(14 ; 18)$ ( $q 32 ; q 21) \operatorname{lgH} / \mathrm{BCL} 2,5.9 \%$ for $\mathrm{t}(11 ; 14)$ (q13;q32) IgH/CCND1, $2 \%$ for $\mathrm{t}(8 ; 14)(\mathrm{q} 24 ; q 32) \operatorname{lgH} / \mathrm{MYC}$ and none for $\mathrm{t}(3 \mathrm{q} 27)$ BCL6 and $\mathrm{t}(11 ; 18)$ (q21;q21) API2/ MALT1.

Conclusion: Cytogenetic analysis has a crescent role in the diagnosis and classification of NHL. In our study the most frequent cytogenetic abnormality was $t(11 ; 14)$ (q13;q32) IgH/CCND1, with age and gender concordant to those described in the consulted literature. 
P57

\section{THE COMMUNITY LABORATORY IN THE EVALUATION OF CARDIOVASCULAR DISEASES}

Gizela Santos, Ana Catarina Guerreiro, João Lago, Renato Lourenço

Laboratório de Análises Clínicas Dr. J. Leitão Santos, Lda. Alverca do Ribatejo.

Introduction: Cardiovascular diseases (CVD) are one of the main morbidity and mortality cause, and their prevalence has been globally rising. Dyslipidemia with high serum values for triglycerides (TRIG) and total cholesterol (CHOL) together with low values for high-density lipoproteins (HDL) are currently used as predictors for CVD.

Goal: Evaluation of TRIG, CHOL and HDL values in serum and calculation of TRIG/HDL and $\mathrm{CHOL} / \mathrm{HDL}$ ratios in a cohort of patients covered by our laboratory.

Methods: A cohort of 22068 patients with requests for $\mathrm{CHOL}$ and $\mathrm{HDL}$ and another of 21729 patients with requests for TRIG and HDL was evaluated during 2017 and segregated by gender (M/F), age, and geographical residence region (estuário do Tejo, Lezíria e Loures/Odivelas). The ratio of $\mathrm{CHOL/HDL}$ was calculated and values higher than 5.0 were considered high risk for CVD. Regarding the TRIG/HDL ratio, values above 2.5 (females) and 3.5 (males) were also considered high-risk. All assays were performed on Roche Cobas 6000 automatic analyzer: CHOL (CHOD-PAP, Roche), TRIG (GPO-PAP, Roche) and HDL (CHER-CHOD-PAP, Roche).

Results: In a total of 22068 patient samples, only 4622 had CHOL/HDL ratio values above 5.0 (13.2\%). Concerning the TRIG/HDL ratio, the total number of analyzed samples was 21279 (9171 male and 12550 female samples), 2510 males (27.3\%) and 3857 females (30.7\%) had ratio values above gender cut-off (respectively 3.5 and 2.5) being considered high-risk for CVD. No significant differences were found relative to geographical area.

Conclusions: Dyslipidemia and CVD are both common in industrialized societies, the community laboratory playing an important role in diagnosis and follow-up of the high-risk population. 
P58

THE ROLE OF THE COMMUNITY LABORATORY ON DIABETES

Ana Catarina Guerreiro, João Lago, Renato Lourenço, Gizela Santos

Laboratório de Análises Clínicas Dr. J. Leitão Santos, Lda. Alverca do Ribatejo.

Goal: Prevalence study of diabetes mellitus on the population covered by a community laboratory.

Methods: A cohort of 23436 patients with glicemia requests was studied during 2017. The results were segregated by gender (M/F), age (from 1 to 101 years old) and geographical residence region (estuário do Tejo, Lezíria e Loures/Odivelas). Criteria used for diabetes are the currently recommended by the Portuguese Health Organization (DGS), i.e., fasting glucose levels $\geq 126 \mathrm{mg} / \mathrm{dL}$ (Hexokinase, Roche). Serum measurements were carried on Cobas 6000 automated analyser (Roche). Patients which exhibited simultaneously diabetes and altered lipid metabolism with total cholesterol > $190 \mathrm{mg} / \mathrm{dL}$ (CHOD-PAP, Roche) were also studied. Data was obtained through the laboratory informatic system (e-deiaLab, Slice) and treated in Excel (Microsoft).

Results: Diabetes prevalence was $8.9 \%$ in the general population, $6.6 \%$ for females, (13762 patients) and $12.1 \%$ for males (9674 patients). Prevalence increases with age, peaking at the interval of 65 to 74 years old. Regarding geographical distribution, Loures/Odivelas presented the highest prevalence (15.4\%). Prevalence for simultaneous diabetes and high cholesterol was $38 \%$.

Discussion: Prevalence of diabetes on the studied population was $8.9 \%$ which is in agreement with the national value of $13.3 \%$ for 2015 (DGS, 2017). Gender differences are clearly apparent, with no significant regional differences. The masculine population has a significantly higher prevalence than the feminine one. The age interval with greater prevalence was the one between ages 65 to 74 years old. One of the main concerns of the Portuguese Plan for Diabetes is centered on prevention and timely treatment in an effort to minimize the later complications of diabetes and providing the whole population with efficient diagnosis and treatment. The community laboratory is, therefore, a very important tool for achieving these objectives. 
P59

URINARY TRACT INFECTION IN A COMMUNITY LABORATORY

João Lago, Ana Catarina Guerreiro, Renato Lourenço, Gizela Santos

Laboratório de Análises Clínicas Dr. J. Leitão Santos, Lda. Alverca do Ribatejo.

Goal: Study the prevalence, evolution and antibiotic susceptibility profiles of the major urinary tract infection agents: Escherichia coli (E.coli), Klebsiella pneumoniae (K.p) and Proteus mirabilis (P.m), over a period of 5 years in a community laboratory.

Materials and Methods: Between January 2014 and December 2018, 32929 midstream urine specimens were collected from outpatients of our laboratory. The culture exams were done by a quantitative method, whereas identification and antimicrobial sensitivity of all strains were carried out by the Vitek 2 system (bioMérieux, Marcy L’Étoile, France) according to the European Committee on Antimicrobial Susceptibility Testing (EUCAST) recommendations.

Results: During this period, the prevalence of urinary tract infection (UTI) was $25.8 \%$ $(\mathrm{N}=8498)$. E.coli $(60.4 \%)$ was the predominant uropathogen isolated, followed by K.p (12.3\%) and P.m (7.6\%). There was a slight increase of the UTI prevalence during the years $(23.9 \%$ in 2014 to $28.6 \%$ in 2018). No trends in prevalence were observed for E.coli and P.m, but for K.p there was a slight increase (10.4\% in 2014 to $13.1 \%$ in 2018). During the study period, high degree of resistance was exhibited to amoxicillin, amoxicillin/clavulanic acid, fluoroquinolones, and cotrimoxazole in almost all strains. In general, E.coli showed less resistant strains than P.m and K.p. E. coli was found to be highly sensitive to nitrofurantoin (97.8\%-98.7\%) and to fosfomycin (97.4\%-98.4\%) during this period. Extended spectrum beta lactamases (ESBL) were evident in K.p and its increase was marked (from $5.7 \%$ in 2014 to $25.5 \%$ in 2018). Carbapenemase producing Enterobacterales (CPE) are a rising problem, especially in $K . p$ (absence in 2014 and 2015 and 3\% in 2018).

Conclusions: E. coli was the major UTI pathogen. High degree of resistance to fluoroquinolones, amoxicillin, amoxicillin/clavulanic acid and cotrimoxazole, shows that the broad use of these drugs needs to be revised, demonstrating its low efficacy as empirical therapy. As documented in the present guidelines, nitrofurantoin and fosfomycin are a good therapeutical option for E. coli UTI, especially in ESBL producers. Multidrug resistance due to production of ESBL or CPE is more frequent in K.p, being an emerging problem of public health, both at the hospital and community levels. 
P60

MOLECULAR BIOLOGY AND MICROSCOPY IN THE DIAGNOSIS OF PLASMODIUM INFECTION IN CENTRO HOSPITALAR UNIVERSITÁRIO DO PORTO

\author{
Faria, João; Mendes, Ana Constança; Santos, Filipe; Freitas, Maria Inês.
}

Laboratory Hematology Unit, Pathology Department, Centro Hospitalar Universitário do Porto, Portugal.

Introduction: Incidence of Malaria in Portugal, has been increasing according to available data (2013 to 2015, Direção Geral de Saúde).

Laboratory methods are crucial to establish the diagnosis and start the appropriate treatment. This includes species identification, in particular of Plasmodium falciparum which can be fatal, and must be differentiated from other species. In addition, species identification of $P$. vivax and $P$. ovale are essential for treatment with primaquine, to eliminate persistent liver stage.

Microscopic examination of peripheral blood is still the gold standard for laboratory diagnosis, however polymerase chain reaction (PCR) methods can be a powerful diagnostic tool, particularly in species identification and confirming infection in cases with negative microscopy but strong clinical suspicion.

The aim of this study was to compare the PCR results, with that of the microscopic examination, in our Hospital.

Materials and Methods: The PCR protocol was developed in house in our Molecular Biology Unit, based on the one originally described by Snounou et al.

In our Hospital this exam is performed when there is a clear need for it, in accordance with close cooperation between clinicians and the pathologists.

Our data includes the cases used for the validation of the PCR technique, and all the subsequent requests, in a period ranging from 2012 to February 2019.

We analyzed 93 samples using both methods and used the Chi-Square for Goodness of Fit test. Discordant cases were further investigated based on clinical data.

Statistic of PCR positives was analyzed according to patient information and country of contact with the parasite.

Results: PCR results found 66 positive and 31 negative samples. In microscopy 56 were positive, 37 negative and in 4 cases it wasn't performed. The $\mathrm{Chi}^{2}$ value was 4,49 and the p-value 0,034 .

$P$. falciparum was identified in 48 cases $(72,7 \%), P$. ovale and malaria in 6 each $(10,6 \%)$ and in 4 cases $(6,1 \%)$ Plasmodium spp was detected without species identification.

Conclusion: As expected $P$. falciparum was the most common species identified. We found no negative PCR results with positive microcopy.

There was good agreement between methods the $p$-value was statically significant at $p<0,05$. In the discordant cases (negative microscopy), half had initiated treatment before blood collection. 
P61

Impact of combined acid-fast bacilli smear microscopy and nucleic acid amplification testing on the sensitivity of diagnosis OF tuberculosis

Óscar Matos, Ana Ferreira, Ricardo Rodrigues, Sandra Paulo, Mariana Martins

Unidade Local de Saúde de Castelo Branco

Introduction: Mycobacterium tuberculosis (MTB) infections remains a significant cause of mortality, despite the evolution of diagnostic and therapeutic strategies for tuberculosis management. A rapid and efficient diagnostic procedure is essential to prevent MTB transmission and enable early disease management. In cases of suspected patients with pulmonary active disease, the Centers for Disease Control and Prevention (CDC) recommends that acid-fast bacilli smear microscopy (AFBSM) should be performed in 3 specimens and suggests, in addition to the former, performing a diagnostic nucleic acid amplification test (NAAT) on the first respiratory specimen, to increase the sensitivity of the diagnostic procedure.

Objectives: We aim to evaluate if our Laboratory results corroborates the CDC guidelines.

Methods: In this study, we retrospectively analyzed the results of combined AFBSM and NAAT performed on respiratory samples during 3 years (2016 to 2018). For each of these samples, AFBSM was performed in our laboratory using a Kinyoun modified Ziehl-Neelsen stain. For the NAATs, the samples were sent to an external laboratory where Xpert MTB/RIF GeneXpert Cepheid ${ }^{\circledR}$ assays where performed. A sample was considered positive if any of these tests detected the infectious agent. False-negatives for AFBSM and NAAT were then counted.

Results: A total of 83 results of combined AFBSM and NAAT in respiratory samples were analyzed, 8 of these samples were positive. We found 1 false-negative AFBSM test and 1 false-negative NAAT.

Discussion and conclusions: The literature reports excellent, up to $100 \%$ sensitivity and $100 \%$ specificity for the Xpert MTB/RIF GeneXpert Cepheid ${ }^{\circledR}$ assay in AFBSM positive specimens and patients without active tuberculosis, respectively. In the NAAT false-negative case it is possible that the bacterial load may have been too low for the assay to detect the bacterial DNA or that AFBSM identified a Mycobacterium other than tuberculosis species bacilli. In the future we would like to compare our AFBSM and NAAT results with culture assay, the gold standard. Nevertheless, our findings suggest that combining AFBSN and NAAT provides the best sensitivity, which corroborates the CDC guidelines. 
P62

\title{
"HAND HYGIENE AND CROSS CONTAMINATION THROUGH PERSONAL USE EQUIPMENT AT THE CHUA CLINICAL PATHOLOGY SERVICE”
}

\author{
B. Gonçalves ${ }^{1}$; A. Guia ${ }^{1}$; M. Fernandes ${ }^{1}$;-I. Casaca ${ }^{1}$; F. Soares ${ }^{1,2}$; J. Matias ${ }^{1,4}$; A. Lapa ${ }^{1,2}$; \\ I. Gago-Rodrigues ${ }^{1,3}$; R. Plácido ${ }^{1,3}$ \\ ${ }^{1}$ Escola Superior de Saúde - University of Algarve \\ ${ }^{2}$ Clinical Pathology Service of Centro Hospitalar Universitário do Algarve - Faro \\ ${ }^{3}$ CES-UAlg - Centro de Estudos em Saúde - University of Algarve \\ ${ }^{4}$ Regional Laboratory of Public Health Dr. Laura Ayres
}

Healthcare-associated infections (HAl) are recognized as a public health problem, being the hand hygiene essential to prevent those infections. In this study, we assess the impact of hand hygiene on cross-transmission of potentially pathogenic microorganisms through the microbiological analysis of healthcare worker's mobile phones. This data was combined with the information provided by a questionnaire to the 14 healthcare workers, in which their knowledge about hand hygiene and the use of mobile phone during the shift was addressed. For the microbiological analysis we collected samples from the mobile phones, before and after shifts, computer mice and keyboards from different sections of the Pathology Service, making a total of 32 samples, followed by the presumptive identification of microorganisms present through morphological, cultural and biochemical characteristics of the colonies. In the microbiological approach we were able to identify linked-coagulase-negative Staphylococci, Methicillin-resistant linked-coagulase-positive Staphylococci, alphahaemolytic Streptococci, Gram-negative and Gram-positive Bacilli and yeasts. We also observe that there is a decrease of microbial growth on the samples after the shifts, comparing with the samples collected before. According to the questionnaire, $92,9 \%$ use the mobile phone during the shift and $57,1 \%$ received training about Hospital Infection Control. However, only $14,3 \%$ can define the five key moments of hand hygiene. We can conclude that most of the volunteers are aware about hands hygiene impact on nosocomial infections spreading. Even though, the most found microorganisms are from the human microbiota, they can have an opportunistic character. Future researches will be important to assure the effectiveness of the measures taken to minimize the mobile phones contamination and to control the HAI. 
P63

CYTOGENETIC ABNORMALITIES (FISH) MOST FREQUENTLY FOUND IN MYELOMA

Naseelah Mussá, Marisa Ferreira, Cristina Duarte

Hospital de Santa Maria - CHULN, E.P.E

Introduction: Plasma cell neoplasms (PCN) result from expansion of a clone of immunoglobulin-secreting, heavy chain class-switched, terminally differentiated $B$ cells. These cells typically secrete a single monoclonal immunoglobulin, an $\mathrm{M}$ protein resulting in the term- Monoclonal gammopathy. Myeloma (M) is a bone marrowbased, multifocal neoplastic proliferation of plasma cells, usually associated with an $M$ protein in serum and/or urine and evidence of organ damage. The diagnosis of $M$ includes many clinical and laboratory criteria. A molecular cytogenetic classification of $\mathrm{M}$ was proposed by the International Myeloma working group placing genetic categories as major indicators of prognosis and forming the basis for risk stratification. The abnormalities detected by Fluorescence In Situ Hybridization (FISH) in PCN are higher than $90 \%$, both numerical and structural in origin.

Goals: To describe the most frequent cytogenetic abnormalities in cases of PCM from 01/01/2016 to $31 / 12 / 2018$ in a tertiary referral hospital.

Materials and methods: data search by Clinidata XXI ${ }^{\oplus}$ Software (Maxdata). Purification of blood marrow samples by immune-magnetic method (RosetteSep). FISH probes used: Vysis ${ }^{\circledR}$ D5S23, D5S721/CEP9/CEP 15 (Hyperdiploidy), Vysis ${ }^{\circledR}$ IGH/FGFR3 DF [t (4;14)], Vysis ${ }^{\circledR}$ IGH/CCND1 XT DF [t(11;14)], XL CDKN2C/CKS1B, Vysis ${ }^{\circledR}$ LSI TP53 (17p13.1).

Results: From the 341 patients studied, 180 were patients with myeloma, 48.9\% females and $51.1 \%$ males. The median of ages was 67.5 (minimal of 30 and maximal of 94). The percentage of myeloma patients with positive results for each of the cytogenetic abnormalities studied were $40 \%$ for $1 q+(1 p 32 / 1 q 21), 20.6 \%$ for Aneuploidies $(5,9,15), 6.7 \%$ for $17 p$ - del $(17 p 13), 10.6 \%$ for $t(4 ; 14)$ and $11.7 \%$ for $\mathrm{t}(11 ; 14)$.

Conclusion: Cytogenetic analysis has a crescent role in the diagnosis and prognosis of the patients with myeloma. In our study the most frequent cytogenetic abnormality detected by FISH was the $1 q+(1 p 32 / 1 q 21)$ which have clinical significance as a poor prognosis feature. 
P64

ACQUIRED HAEMOPHILIA A - INSIDE THE BLEEDING...

Naseelah Mussá, Marisa Ferreira, Calisto Carina, Carriço Fátima

Hospital de Santa Maria - CHULN, E.P.E

Introduction: Acquired hemophilia $(\mathrm{AH})$ is a rare but potentially life-threatening bleeding disorder caused by the development of autoantibodies (inhibitors) against plasma coagulation factors, most frequently against factor VIII - acquired haemophilia A (AHA). Most cases occur in older adults and equally in both genders. Various conditions have been associated with AHA (pregnancy, infections, malignancies, advanced age) but, approximately $50 \%$ of cases are idiopathic. The clinical signs and symptoms of acquired hemophilia differ from those of hereditary hemophilia. Most patients with AHA present with skin, muscles, soft tissues or mucous membranes hemorrhages and no hemarthrosis. The diagnosis is difficult, because the condition is rare and the patient does not have a personal or family history of bleeding disorders. Laboratory study include platelet count, prothrombin time (PT)-usually normal, the activated partial thromboplastin time (aPTT) prolonged (not reversed on a correction study), reduced FVIII levels and evidence of an FVIII inhibitor. Other factor levels are determined to establish inhibitor specificity and once the acquired inhibitor is detected, it should be quantified to access the severity of the disorder and the risk of hemorrhagic complications.

Case study: 79 year old female with a large spontaneous hematoma on the left thigh with three days evolution and marked edema of the left limb. The patient reported an episode of difficult controlling epistaxis three weeks before the actual symptoms, treated in an emergency room. Denied fever and other symptoms. The clinical course evolved with dysphonia due to an extensive spontaneous hematoma in the cervical area and bruising in the upper limbs. Evaluation revealed a cervical adenopathy.

Laboratorial tests: Hgb - 6.5 g/dl, PLT-233×109/L, PT - 10.8s (INR-0.93), APTT->100s, Fibrinogen: $387 \mathrm{mg} / \mathrm{dl}$, D-Dimer: $0.60 \mathrm{ug} / \mathrm{ml}$. Factor VIII C: $<1 \%$, factor IX C: $65 \%$, factor VIII inhibitor: positive (471 Bethesda units). Rheumatoid factor-negative.

Discussion: A multidisciplinary approach is essential in the diagnosis and management of AHA. The search for an implicated co-morbidity is always advised. Low levels of factor VIII C and high levels of inhibitor are all considered markers of severity, prognosis and treatment response. 
P65

NON-HODGKIN'S LYMPHOMA CHANGES IN VOLUME, CONDUCTIVITY AND SCATTER

Bárbara Abreu; Jorge Reis; Micaela Batista; Hugo Macedo; Ana Paiva; Joana Diamantino; Frederico Valido

Clinical Pathology Department - Instituto Português de Oncologia de Coimbra

Introduction: Non-Hodgkin's Lymphoma (NHL) accounts for about $2.3 \%$ of all neoplasms and may derive from abnormal and malignant clonal proliferation of $B$ or T lymphocytes, with increasing frequency with advanced age and diverse etiology. Automated cell analyzers are basic hematology equipment that allow rapid and reliable analysis of peripheral blood. They characterize white blood cells using parameters such as volume, conductivity and scatter that may be altered for lymphocytes in NHL patients.

Objective: With this work we intended to evaluate possible changes in volume, conductivity and scatter in lymphocytes from patients with different types of NHL.

Material and methods: In this study, $307 \mathrm{NHL}$ patients were included, and volume, conductivity and scatter (VCS) data of each type of lymphoma registered, as well as the total amount of leucocytes and lymphocytes, from 2014 to 2018. Statistical analysis was performed using SPSS $\left(\right.$ IBM $\left.^{\circledR}\right)$. Student T Test was used to compare the variables of the patient group with control group. One-way ANOVA test was applied to compare different NHL types relative to the VCS parameters and with the control group.

Results: In our sample the most frequent lymphoma was lymphocytic lymphoma (28.7\%), followed by mantle cell lymphoma (25.1\%) and follicular lymphoma (13\%). From the parameters evaluated, conductivity showed highest differentiation between control group and patient group $(p<0.0001)$ and among NHL patients with greater emphasis for Marginal Zone Lymphoma in relation to other lymphomas. Statistical difference between control group and patient group was also obtained for scatter ( $p=$ 0.027).

Conclusions: We can therefore conclude that in relation to VCS parameters, conductivity and the scatter showed variation between the different lymphomas evaluated. Variation that was also observed between the total group of patients and the control group. Conductivity was the parameter that suffered highest variations between the different lymphoma groups. 
P66

ROUTINE DETERMINATION OF HEMOGLOBINS A2 AND F, WHEN PERFORMING GLYCATED HEMOGLOBIN TEST BY HIGH PERFORMANCE LIQUID CHROMATOGRAPHY

Ana Isabel Ferreira, Sandra Paulo, Óscar Matos, Ricardo Rodrigues, Mariana Martins

Serviço de Patologia Clínica da Unidade Local de Saúde de Castelo Branco

Introduction: Hemoglobinopathies are hereditary diseases characterized by mutations of globins genes, leading to quantitative changes in the synthesis $(\alpha / \beta$ thalassemia) or to the formation of structurally abnormal hemoglobin $(\mathrm{Hb})$ variants. The clinical presentation varies from mild anemia to severe transfusion-dependent disease. These conditions represent an important public health issue, so the earliest diagnosis is fundamental to reduce morbidity and mortality. The most common hemoglobinopathies in Portugal are Drepanocitosis ( $\mathrm{Hb}$ variant - $\mathrm{HbS}$ ) and $\beta$ Thalassemia. $\mathrm{Hb}$ is constituted by 4 globin chains, in adult life normally presented as $\alpha_{2} \beta_{2}(\mathrm{HbA}), \alpha_{2} \Upsilon_{2}(\mathrm{HbF})$ and $\alpha_{2} \delta_{2}(\mathrm{HbA} 2)$, that can be quantified by different laboratory techniques.

Description - Results: When processing the samples for determination of glycated hemoglobin $(\mathrm{HbA} 1 \mathrm{c})$ as part of the Diabetes Mellitus follow-up, our automated analyzer also performed the determination of $\mathrm{HbA} 2(<3,5 \%)$ and $\mathrm{HbF}(<1,8 \%)$. When a percentual elevation in the values was observed, we notified the responsible physician. The aim of this retrospective analysis was to characterize the $\mathrm{HbA} 2$ and $\mathrm{HbF}$ results in 2018, and verify if any further diagnostic study was done. The samples (plasma-EDTA) were processed by High Performance Liquid Chromatography (HPLC) ADAMS $^{\mathrm{TM}}$ A1C HA-8160. We processed 5305 samples corresponding to 3752 patients, age: 1 to 101 years; 1194 men and 2558 women. 58 of these patients ( 34 men and 24 women) had results above the cut-off defined in our Laboratory: 28 (HbA2 $\geq 3,5 \%), 27$ $(\mathrm{HbF} \geq 1,8 \%)$ and $3(\mathrm{HbA} 2 \geq 3,5 \%$ and $\mathrm{HbF} \geq 1,8 \%)$. We could only confirm further diagnostic study in one of these patients.

Discussion: This exam was ordered as part of the Diabetes Mellitus protocol of our Institution, so the altered results were occasional findings. It would be interesting to continue this analysis trying to correlate our results with previous diagnosis, or clinical signs and symptons of these patients that may be compatible with these diseases. $\beta$ thalassemia leads to a decrease of $\beta$ globin chain, with increase more pronounced of $\mathrm{HbA} 2$ (minor form) and $\mathrm{HbF}$ (intermedia form). Carriers (heterozygotes) may be asymptomatic, but there is a $25 \%$ probability of progeny with a more severe disease, so diagnosis confirmation and genetic counseling is relevant. 

VARIATION.

Pinheiro Jorge', Alvim A. , Anastácio M.; Costa N., Caeiro J., Cardeira S., Carriço A., Carvalho A., Cunha R., Ervilha C., Ferreira F., Figueiredo M., Gomes D., Lopes C., Louro N., Menezes M., Mendonça A., Oliveira A., Órfão D., Pedrosa J., Pires Letícia., Santos L., Santos M., Simões I., Vieira R., Silva C., Paquim A., Ostapenko N., Castro Ricardo

Pathology Service of Centro Hospitalar de Leiria EPE

\section{Introduction:}

Biological variation is widely used has a reference for analytical performance specification in clinical laboratories. In a previous work (1), we suggested that the patient's distribution data charts (PDC), could be used as model $1 \mathrm{~b}$ analytical performance specification method of the 1st Strategic Conference Consensus of EFLM, to simulate if an allowable error affects or not the patient's results.

\section{Objective:}

To show that, when the laboratory defines a maximum uncertainty measurement under the within-subject biological variation known for a parameter (CVi), the patient' results will not be significantly affected by errors smaller than the upper limits of the quality control.

\section{Material and Methods:}

Creatine kinase (CK) was used as the model and Modulab Gold was used as the source for its PDC. According to the European Biological Variation Study (EuBIVAS) (2), with $95 \%$ confidence intervals $(\mathrm{CI})$, the within-subject biological variation (CVi) for $\mathrm{CK}$ is $14,5 \%$.

We defined the maximum allowable uncertainty of measurement for CK as CVmax = $8 \%$, with a $1 / 2$ s exclusion rule.

\section{Results:}

A bias of $+8 \%$, visible on the quality control charts for CK due to a technical error, did not show any shift on the patient's distribution data (PDC).

\section{Conclusion:}

When a clinical laboratory implements a quality control program (QCP) with a maximum allowable uncertainty measurement under the CVi of a biological magnitude, any technical error within these limits will be absorved by the biological variation, and will "do no harm" to the patient, because it will fall within the analytical performance specifications 
1"A simulation method for investigating the impact of analytical performance on the probability of patient outcomes? About a case on a Hospital Center's laboratory", Pinheiro J, Pinheiro Jorge, Paquim Ana, Ostapenko Nataliya, Pinto Ana Bento, Castro Ricardo, in "10th National Congress of the Portuguese Society of Clinical Chemistry, Genetics and Laboratory Medicine", Clin Chem Lab Med 2018; 56(11): eA171-eA202 "Harmonization initiatives in the generation, reporting and application of biological variation data", Aasne K. Aarsand*, Thomas Røraas, William A. Bartlett, Abdurrahman Coşkun, Anna Carobene, Pilar FernandezCalle, Niels Jonker, Jorge Díaz-Garzón, Federica Braga and Sverre Sandberg, on behalf of the European Federation of Clinical Chemistry and Laboratory Medicine (EFLM) Working Group on Biological Variation, Clin Chem Lab Med 2018 
P68

NEED FOR SPEED - SCREENING OF CARBAPENEM-RESISTANT ENTEROBACTERIACEAE IN A TERTIARY HOSPITAL

Carolina Queiroz, Catarina Chaves, Fernando Rodrigues

Serviço de Patologia Clínica, Centro Hospitalar e Universitário de Coimbra

Introduction: Rising numbers of Carbapenem-resistant Enterobacteriaceae (CRE), fostered by the lack of preemptive screening of patients in healthcare facilities, have become a public health concern. This problem has led national health entities in Portugal (PPCIRA) to publish recommendations for prevention of CRE transmission in acute care hospitals.

Material And Methods: Review of the screening program for CRE implemented at our hospital to identify asymptomatic carriers and implement containment measures, and of its results, during the period between Jan 2017 and Feb 2019.

Results: Given the relationship between identification of CRE carriage and placement of effective infection control interventions, a rapid molecular-based screening method with the ability to test directly from clinical specimens (rectal swabs) without need for culture (Xpert Carba-R, Cepheid) was selected by our hospital. Screening is performed to target risk populations, as defined by PPCIRA, and to patients admitted to selected wards, such as Hematology, Transplant, Burn and Intensive Care Units. Upon an initial negative result, it is recommended to perform a second confirmatory swab within 48 hours. A total of 3126 tests were performed to 2116 patients - $55.8 \%$ males and $44.2 \%$ females with a mean age of 66 years. 2254 screening tests and 872 confirmatory tests were performed with confirmatory tests being performed in $44.2 \%$ of the negative cases, and an overall CRE positivity rate of $10.7 \%(n=334)$. The resistance genes identified were mostly KPC (94.4\%; $n=319)$, with only $4.4 \%$ VIM and $0.9 \%$ of OXA-48 and no IMP1 or NDM cases.

Conclusion: National recommendations define target risk populations and frequency of testing but leave the preferred test method open to debate. The molecular based approach selected might be costly compared to other alternatives but has the advantage of direct testing and rapid turnaround times, reducing the need of isolation beds for cases pending confirmation of results in a high CRE prevalence area. Also, longer turnaround times of the culture-based methods can carry other direct and indirect costs. Effective use of this technology depends on close coordination between clinical microbiology and infection control teams, and measures to raise the percentage of confirmatory tests performed are needed in our hospital. 
P69

HEREDITARY HEMOCHROMATOSIS: FREQUENCY OF RELATED GENE MUTATIONS

M. Teresa Rodrigues, Nuno Costa e Silva, Margarida Lourenço, M. Fátima Martins, Fernando Rodrigues

Serviço de Patologia Clínica, Centro Hospitalar e Universitário de Coimbra

Introduction: Hereditary hemochromatosis $(\mathrm{HH})$ is an inherited autosomal recessive disorder of iron metabolism. Due to excessive absorption, iron accumulates in the liver, heart and other organs resulting in damage to their structure and function. It is one of the most common genetic diseases in Caucasians with a prevalence of nearly 1 in 300. Molecular genetic testing for hereditary hemochromatosis is recognized as a reference test to confirm the diagnosis of suspected $\mathrm{HH}$ or to predict its risk. The majority (>90\%) of patients with clinically characterized $\mathrm{HH}$ are homozygous for the $\mathrm{C} 282 \mathrm{Y}$ variant in the HFE gene. Two other mutations have been described, H63D and $\mathrm{S} 65 \mathrm{C}$, associated with a milder form of the disease. The aim of this study is to investigate the frequencies detected for $\mathrm{C} 282 \mathrm{Y}, \mathrm{H} 63 \mathrm{D}$ and $\mathrm{S} 65 \mathrm{C}$ mutations in patients from our hospital to whom the genetic study of hemochromatosis was requested from 2016 to the present date (end of February 2019).

Materials and methods: The study of the presence of mutations C282Y, H63D and S65C in HFE gene in our hospital was made by reverse hybridization using "Haemochromatosis StripAssay B VIENNA LAB" kit.

Results: Of the 221 patients in our sample 52,94\% showed no mutation and 47,06\% some type of mutation: 7,69 \% exhibited C282Y heterozygous mutation (C282Y/WT); 1,81\% C282Y homozygous mutation; 25,34\% H63D heterozygous mutation (H63D/WT); 5,88\% H63D homozygous mutation; and 3,17\% had $\mathrm{C} 282 \mathrm{Y} / \mathrm{H} 63 \mathrm{D}$ heterozygous mutation. As for the S65C mutation $2.26 \%$ of the patients had an heterozygous mutation (S65C/WT), 0,45\% had H63D/S65C heterozygosity and 0,45\% had C282Y/S65C heterozygosity .

Conclusion: The mutation most frequently associated with hemochromatosis (C282Y homozygous mutation) was only found in $1,81 \%(n=4)$ of our patients, being the most common mutation present in our study the H63D heterozygous mutation $(25,34 \%$, $\mathrm{n}=56$ ). The absence of any mutation is seen in over half of the patients studied $(52,94 \%, n=117)$. 
P70

PERFORMANCE OF PNAEQ PARTICIPANTS IN EXTERNAL QUALITY ASSESSMENT IN PREANALYTICAL PHASE - 2018

\author{
Catarina Ventura ${ }^{1}$, Silvia Viegas $^{1}$, Ana Cardoso $^{1}$, Helena Correia ${ }^{1}$, Ana Faria ${ }^{1}$ \\ Instituto Nacional de Saúde Dr. Ricardo Jorge, Departamento de Epidemiologia, Unidade de \\ Avaliação Externa da Qualidade ${ }^{1}$
}

Introduction: The National Program of External Quality Assessment (PNAEQ) provide tools for pre-analytical phase, allowing detection, monitoring and reduction/elimination of errors that could affect the reliability of the laboratory results.

Aim: Performance of PNAEQ participants in External Quality Assessment in preanalytical phase during 2018.

Material e Methods: PNAEQ promoted an annual meeting with the Working Group of pre-analytical phase to discuss the results from the last year and to collect suggestions to implement in next year scheme. The scheme of 2018 included: 1) quality indicators; 2) internal audit; 3) mystery client (patient and clinician); and 4) training actions. Were sent to participants general and individual evaluation reports.

Results: In 2018, there were 16 participants registered in pre-analytical phase scheme. The participation rate and the performance results for each trial were:

1) Quality indicators: 67\% of participation. "Registration Errors" was the indicator with the highest average (1,2\%).

2) Internal audit: $60 \%$ of participation. The most critical results were for "What is the collection system used?" (48\% Open / 52\% Closed) and "Did you use a needle with a security system?" (Yes 42\% / No 58\%).

3) Mystery client: $75 \%$ of participation. Mystery client "patient" with $43,4 \%$ of discrepancy between responses (83\% in "Collection Instructions"). Mystery client "clinician" with 16,5\% of discrepancy between responses (25\% in "Parameters determined in laboratory or subcontracting").

4) Training actions: 40 participants. Themes presented: Potassium and Pre-analytical - case study; Pre-analytical in Microbiology; Preparation and interpretation of Risk Matrix in Pre-analytical; Uncertainties associated with variability of Pre-analytical phase.

Discussion e Conclusion: The results of EQA Pre-analytical scheme can be used as scientific evidence for monitoring the laboratory processes, detecting errors, analysing the causes of the increase of errors and implementing preventive and corrective actions. We suggest the participants to a) hold a regular cases discussion; b) promote collaborators training to reduce registration errors and the instructions inconsistency c) implement a closed system for blood collection, and d) use of security system needles. 